\title{
Brownian Motion in the Fluids with Complex Rheology
}

\author{
V. V. Rusakov ${ }^{1,2}$, Yu. L. Raikher ${ }^{1,2} *$, R. Perzynski ${ }^{3}$ \\ ${ }^{1}$ Institute of Continuous Media Mechanics, Russian Academy of Sciences, \\ Ural Branch, 1 Korolyov street, Perm, 614013, Russia \\ ${ }^{2}$ Perm National Research Polytechnic University, 29a Komsomol avenue, 614990, Perm, Russia \\ ${ }^{3}$ Université Pierre et Marie Curie - PHENIX, UMR 8234, 4 place Jussieu, 75005 Paris, France
}

\begin{abstract}
Theory of Brownian motion of a fine particle in a viscoelastic fluid continuum is developed. The rheology of the embedding medium is described in terms of classical structure (spring-and-damper) schemes. It is shown that a great variety of conceivable ramifications of such structures could be reduced to an effective Jeffreys model: a Maxwell chain shunted by a damper. This scheme comprises just three material parameters: two for viscosities (fast and slow) and one for elasticity. For the thermal motion of a particle in such a fluid, the set of Langevin equations is derived. In the cases of $1 \mathrm{D}$ translational and $2 \mathrm{D}$ orientational (rotation about fixed axis) motions, the time dependencies of mean-square displacements are found analytically. It is shown that in a Jeffreys fluid, the Brownian motion has three regimes: fast diffusion (short times), slow diffusion (long times) and a crossover of those resulting in virtual localization (dynamic confinement) of the particle. Experimental evidence for that taken from the literature is presented. The developed formalism is applied to the particles with "frozen-in" dipole moments, whose orientational motion is a combination of Brownian diffusion and regular excitation by an AC field. The dynamic susceptibilities are calculated for the ensembles of particles liable for either 2D or 3D rotations. Comparison shows that the out-of-phase part of the susceptibility $\chi^{\prime \prime}(\omega)$ in 3D case differs considerably from that of $2 \mathrm{D}$ case. Function $\chi(\omega)$ for the forced 3D rotary diffusion in a Jeffreys fluid is found for the first time. In our view, it would be useful for realistic theoretical interpretation of microrheology data and the more so for estimating the particle-mediated AC field-induced heating (magnetic hyperthermia) in viscoelastic fluid media.
\end{abstract}

Keywords and phrases: Brownian motion, viscoelastic media, kinetic equation, stochastic equations, microrheology, magnetic particles

Mathematics Subject Classification:

\section{Introduction}

Thermofluctuational diffusion motion of fine particles embedded in complex fluids is the subject of intense and yet increasing interest. Brownian motion, being a ubiquitous process, is very important from the fundamental as well from practical viewpoints. Diffusion of micro- and/or nanosize particles plays

${ }^{*}$ Corresponding author. E-mail: raikher@icmm.ru, yuriy.raikher@gmail.com

(C) EDP Sciences, 2015 
essential role in the physical chemistry, chemical technology, and material science of inorganic composites and polymers. Diffusion-mediated transport is an inherent part of cell and tissue biology and biotechnology, while diffusion-aided drug penetration is a vital subject in medicine. All this variety of processes dwells nowadays in need for the theory of Brownian motion beyond the classical limit, where the molecules/particles move through a linearly viscous (Newtonian) fluid. This need is at least threefold. First, such a theory can provide a more detailed description of the molecule and nanoparticle penetration through complex systems of inorganic, organic and biological origin. Second, if to use the Brownian particles as microprobes, as it is done in micro- and nanorheology [1-4], the theory shows the way to extract from the recorded data a reliable knowledge on the scale-sensitive mechanical properties of the tested media in a wide frequency band. Third, the theory is necessary for analyzing dissipation due to the forced motion of the particles in their complex fluid environment.

The demand for extended knowledge of the power losses comes from the fast developing interdisciplinary area of magnetic hyperthermia $[5,6]$. This method, is aimed at the fight against malignant (e.g. oncological) cells. It exploits the heat generated by AC magnetic fields in a tissue filled with ferromagnet-containing nanoparticles. This externally targeted and controlled heat injection might be used either directly (since the bad cells are more sensitive to temperature enhancement that the normal ones) or indirectly, e.g. for acceleration of pharmacokinetics of the drugs brought in on the surface of nanoparticles or inside the magnetic nanocapsules [7-9], microspheres, etc. In the considered situation, the heat is generated by two coupled mechanisms. One is due to relaxational motion of the magnetic moment inside the particle body. The other originates from the viscous friction, which accompanies mechanical motion of the particle with respect to the surrounding medium. Given the small (micro or nano) size of the particles involved in all the above-mentioned applications, it is clear that any forced their motion, if any, is always overlaid by a stochastic process, viz. Brownian diffusion.

The prime concern of the hyperthermia technique of any type, is its optimization $[5,10,11]$, i.e., maximizing the specific heat generation (absorption power) in a given environment not exceeding the phisiological limits of electromagnetic exposure. The magnetic (intraparticle relaxation) contribution to the absorption has been studied to fine details for a variety of ferromagnet and ferrite nanoparticles [1012]. The rheological (outer friction) part of the field-induced dissipation, is known to much less extent. Meanwhile, this contribution is equally important for optimization [13], and it is the more so given the diversity of soft matter matrices, which the particles could be embedded in.

The so-called "active" modifications of micro/nanorheology $[14,15]$ and the hyperthermia approach have very much in common. Indeed, in both situations on the Brownian particles possessing e.g. magnetic dipoles, an external driving field is imposed. Usually, this is done not with a single object but with an ensemble of particles that admits statistical treatment. The field-induced responses, one timemechanical and/or magnetic (as for microrheology), and the other time - thermal (as for hyperthermia), are measured, analyzed and utilized.

It is well known, that Brownian motion, both free and forced, in non-Newtonian (viscoelastic) fluids differs considerably from that in linearly viscous (Newtonian) ones. This qualitative observation is apparent and hardly needs any proofs. However, as to the quantitative theory of Brownian motion in viscoelastic media, it is developing quite slowly. To a good part, this is due to the diversity of the fluids generally called viscoelastic. Indeed, an inorganic surfactant solution and the amebae cytosol seem to have very little in common. That is why, the developed theoretical models are usually particularly oriented, and seeking of universality is not their prime objective.

In our work, which in a way is a review, we try another approach. The rheological models applied to the viscoelastic medium are classical. They are taken from conventional phenomenology and, thus, comprise minimal number of material parameters. The advantage is that on this basis one can build up a consistent theory logically "branching off" the well-known Newtonian case. Being not that detailed, such approach, however, profits in full from the advantages of phenomenology: simplicity and robustness. Besides, it is easily extendable for a number of rheological schemes, where the parameters are more numerous, that is, better adjusted to account for specific real cases. On the other hand, being as simple as it is, the theory 
is limited to some domain of viscoelasticity. For example, it cannot account for the cases dominated with fractional diffusion. However, within the range of its applicability, our model provides a useful and handy framework for interpreting the stochastic behavior of particles in strongly viscoelastic fluids.

The paper is organized as follows. In Section 2, conventional rheological schemes used in macroscopic phenomenology are described and classified. As shown, all of them could be reduced to an effective Jeffreys (three-parameter) fluid medium. In Section 3, the set of Langevin equations and the pertinent kinetic (Fokker-Planck) equation are derived for a particle embedded in a fluid with Jeffreys rheology. Section 4 is focused on investigation of 1D translational Brownian motion in this fluid, and, as an example, the ability of the model to account for the free particle diffusion inliving polymer systems is demonstrated. In Section 5, the theory is extended to the case of a particle with a finite mass. One of the gains of this description, is that it helps to reveals the origin of unphysical predictions occurring in the case when the Maxwell model is used instead of the Jeffreys one. In Section 6, the problem is transformed from Brownian 1D translational motion to Brownian rotation about fixed axis. In the latter case, the reduced phase space implies that the rotating particles are disk-like (planar rotators). The mean-square angle fluctuation for them is evaluated and analyzed. The problem pertinent to active (magnetic) nanorheology and hyperthermia is studied in Section 7 for the same planar rotator (2D) model. There the dynamic susceptibility of a statistical ensemble of non-interacting rotators with "frozen-in" magnetic moments is obtained via the linear response theory. In Appendices I and II it is shown, how this result could be re-derived with different methods. The purpose is that the analytical procedures, which easily yield magnetic susceptibility in the case of rotators, pose huge calculational difficulties when the particles are given full orientational freedom, i.e., the rotations are 3D. It turns out that only by constructing the set of orthogonalized statistical moment equations directly from the Langevin set - this procedure is proposed and tested in Appendix II - one can put the dynamic magnetic susceptibility of an ensemble of 3 D rotating particles in a Jeffreys fluid to a plausible analytical form. This is done in Section 8, where the magnetic spectra, i.e., imaginary components of the dynamic magnetic susceptibility are compared for $2 \mathrm{D}$ and $3 \mathrm{D}$ cases. It turns out that the planar rotator $(2 \mathrm{D})$ model, although providing general qualitative resemblance, in quantitative aspect is rather far from the results given by the 3D treatment. In particular, this concerns the lineshapes of the magnetic absorption spectra. In Conclusions, the finding of the work are summarized.

\section{Classical models of the fluids with complex rheology}

In our view, a simple and quite fruitful way to deal with this set of problems is provided by modelling the carrier fluid, where the particle moves, with the aid of the rheology schemes consisting of two conventional "building blocks", namely, a damper (viscous friction) and a spring (restoring force) [16,17]. As to the particles, they are assumed to be of micron size or smaller, and spherical in shape. Their motion rates are as low as to enable one to reduce the hydrodynamics to the Stokes limit [18]. Therefore, the action of the two basic elements presenting the microsphere environment is reduced to the viscous and elastic forces as

$$
\begin{aligned}
& f_{\eta}=-\zeta \dot{x}, \zeta=6 \pi \eta a, \\
& f_{\kappa}=-\kappa x, \kappa=6 \pi G a .
\end{aligned}
$$

Here $a$ is the particle radius, $\eta$ viscosity coefficient, and $G$ shear modulus; $\zeta$ and $\kappa$ the friction and elasticity coefficients of the particle, respectively. The negative signs in right-hand sides of Eqs. (2.1) mean that both forces are directed opposite to the particle motion thus impeding it. We remark that according to a commonly used hypothesis, the viscous and elastic coefficients are based on one and the same Stokes formfactor $6 \pi a$, see [1], for example.

The focus of interest here is the fluctuational motion of a micro- or nanoparticle inside a complex fluid whose rheology could be described by an arbitrary combination of the two above-named basic elements. The general theory of equilibrium thermal fluctuations in dynamic systems has a long story dating back to the classical Einstein's work [19]. In modern literature there is a number of books on the subject [2024]. Looking into it, one finds out that there exist two equally fundamental approaches. One, historically 
the first, developed by Einstein [19] uses a diffusion-type kinetic equation. The second, proposed by Langevin [25], is based on the set of stochastic equations. For a detailed historical review on the theory developments, see [24].

In either of these approaches, the action of thermal fluctuational on a particle is modelled by a set of random forces. This is virtually trivial for the case of a linearly viscous (Newtonian) fluid but makes a problem when the rheological scheme is multi-element. In below, using a number of examples, we show how an arbitrary rheological scheme of a complex fluid, which, generally speaking it not fit for introducing random forces, could be turned to a form well enabling consistent Brownian-motion treatment. Mathematically, this means re-formulation of the particle dynamics as a set of first-order (with respect to time) linear ordinary differential equations (LODE).

The rheological schemes considered here obey certain simple rules. The force is constant along any sequential chain of elements. For a parallel configuration of the elements, the reaction forces sum up [8]. Therefore, any sequence of elements (elastic or viscous) may be replaced by a single effective element of the same type. Under a constant force the net displacement (the ends of the chain) is the sum of all the partial displacements so that the effective friction and elasticity coefficients of the chain combine as

$$
\begin{aligned}
& \left(\zeta_{\text {eff }}\right)_{\text {seq }}^{-1}=\left(\zeta_{1}\right)^{-1}+\left(\zeta_{2}\right)^{-1}+\ldots \\
& \left(\kappa_{\text {eff }}\right)_{\text {seq }}^{-1}=\left(\kappa_{1}\right)^{-1}+\left(\kappa_{2}\right)^{-1}+\ldots
\end{aligned}
$$

For the case of parallel connection of similar elements, i.e., if they resist in coherence, then the forces sum up, while the displacement is common. In result, several elements of the same kind may be replaced by an effective one according to the rule

$$
\begin{aligned}
& \left(\zeta_{\mathrm{eff}}\right)_{\mathrm{par}}=\zeta_{1}+\zeta_{2}+\ldots, \\
& \left(\kappa_{\mathrm{eff}}\right)_{\mathrm{par}}=\kappa_{1}+\kappa_{2}+\ldots
\end{aligned}
$$

The simplest rheological scheme that models an elastoviscous medium was proposed by Kelvin, see Fig. 1. It consists of an elastic and viscous elements set in parallel. Such a pattern may be considered as a primitive model of a particle in a gel. Such a particle "remembers" its initial position, and re-enters it as soon as the forces cease to act. The equation of the particle motion in this case is

$$
m \ddot{x}=-\zeta \dot{x}-\kappa x \text {. }
$$

Reduction of this equation to the equivalent LODE form is trivial:

$$
\dot{x}=v, \quad m \dot{v}=-\zeta v-k x .
$$

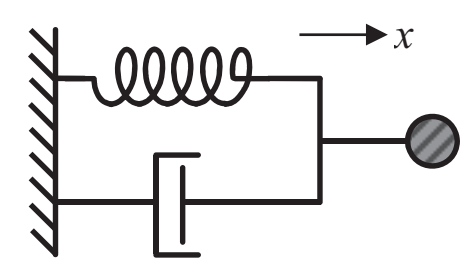

Figure 1. Kelvin rheological scheme.

The basic scheme to resemble a viscoelastic fluid is Maxwell's model, where the elastic and viscous elements are connected sequentially, see Fig. 2. The resistance force in the primitive Maxwell chain is constant, the displacements sum up:

$$
\left\{\begin{array}{c}
f_{1}=f_{2}=f ; \quad x=x_{1}+x_{2} \\
f_{1}=-\zeta_{M} \dot{x}_{1} ; f_{2}=-\kappa_{M} x_{2} .
\end{array}\right.
$$




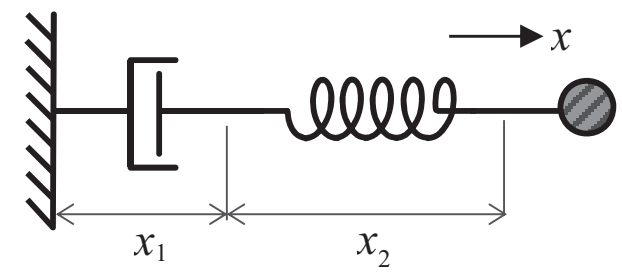

Figure 2. Maxwell rheological scheme.

The reaction force of the viscous element is

$$
f_{1}=f=-\zeta_{M}\left(\dot{x}-\dot{x}_{2}\right) .
$$

Eliminating from (2.7) the velocity of the elastic element and differentiating the elastic force, one gets the resistance force of the Maxwell chain:

$$
\left(1+\tau_{M} \frac{\partial}{\partial t}\right) f=-\zeta_{M} \dot{x}, \quad \tau_{M} \equiv \frac{\zeta_{M}}{\kappa_{M}} .
$$

This equation shows that to fast motions, i.e., $\tau_{M}(\partial f / \partial t) \gg f$, the fluid responds in elastically: $f \simeq-\kappa_{M} x$. In the opposite limit, when the motion is slow, the response force is mostly viscous: $f \simeq-\zeta_{M} \dot{x}$. The reference time $\tau_{M}$ that separates the limiting regimes is an important material parameter; for real systems it varies in the range $10^{-2}-10^{3} \mathrm{~s}[16]$. The equations of motion for this case presented in the standard form are

$$
\left\{\begin{array}{l}
\dot{x}=v, \\
m \dot{v}=f, \\
\dot{f}=-\gamma_{M} f-\kappa_{M} v, \gamma_{M} \equiv 1 / \tau_{M} .
\end{array}\right.
$$

Apparently, parallel connection of several Kelvin chains yields a single effective Kelvin element with the parameters (2.3). Similarly, setting several Maxwell elements sequentially, one gets a single effective Maxwell chain with the parameters (2.2).

Let us now construct a standard form for the scheme, where two Kelvin chains are connected sequentially, see Fig. 3, left pane. According to the above-presented rules, one has
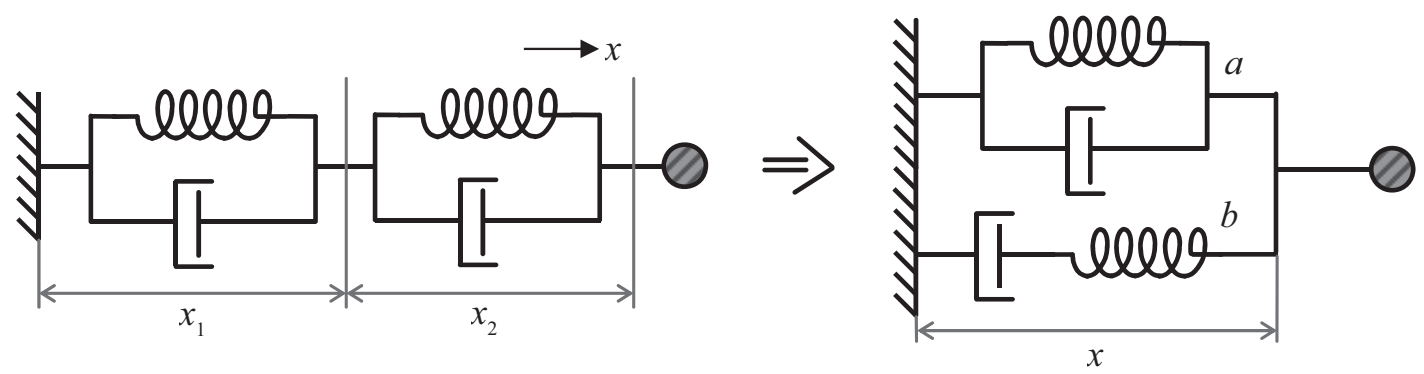

Figure 3. Sequential connection of two Kelvin chains (left) and its equivalent LODE scheme (right).

$$
\left\{\begin{array}{l}
f_{1}=f_{2}=f, x=x_{1}+x_{2} \\
f_{\alpha}=-\kappa_{\alpha} x_{\alpha}-\zeta_{\alpha} \dot{x}_{\alpha} \equiv-\kappa_{\alpha} \hat{R}_{\alpha} x_{\alpha}, \alpha=1,2 .
\end{array}\right.
$$


Hereafter we use short notation for the retardation operator and introduce pertinent reference times:

$$
\hat{R}_{\alpha} \equiv 1+\tau_{\alpha} \frac{\partial}{\partial t}, \quad \tau_{\alpha} \equiv \frac{\zeta_{\alpha}}{\kappa_{\alpha}}
$$

Eliminating displacement $x_{1}$ from set (2.10) yields the force

$$
f=-\kappa_{1} \hat{R}_{1}\left(x-x_{2}\right) .
$$

Subjecting it to operator $-\kappa_{2} \hat{R}_{2}$ and taking into account that the force for chain 2 is

$$
f=-\kappa_{2} \hat{R}_{2} x_{2}
$$

one gets the response force of the whole scheme

$$
\left(\kappa_{1} \hat{R}_{1}+\kappa_{2} \hat{R}_{2}\right) f=-\kappa_{1} \kappa_{2} \hat{R}_{1} \hat{R}_{2} x .
$$

With allowance for definition (2.11), this equation takes the form

$$
\left[\kappa_{1}+\kappa_{2}+\left(\zeta_{1}+\zeta_{2}\right) \partial_{t}\right] f=-\left[\kappa_{1} \kappa_{2}+\left(\zeta_{1} \kappa_{2}+\zeta_{2} \kappa_{1}\right) \partial_{t}+\zeta_{1} \zeta_{2} \partial_{t}^{2}\right] x
$$

As seen, this equation does not have standard form. To proceed, we note that the passage from the left end of the scheme Fig. 3 to its right one might be done along any of three ways:

- via the sequentially connected viscous elements,

- via the sequentially connected elastic elements,

- via the two Maxwell chains.

Therefore, the standard rheological scheme corresponding to the initial one consists of the parallel connection of Kelvin (a) and Maxwell (b) chains, see Fig. 3, right pane. Accordingly, the response force of the equivalent scheme is

$$
f=-\zeta_{a} \dot{x}-\kappa_{a} x+f_{b}
$$

Substituting this relation in Eq. (2.15), one gets the equation for the new response force, $f_{b}$ :

$$
\begin{gathered}
\left(\kappa_{\Sigma}+\zeta_{\Sigma} \partial_{t}\right) f_{b}-\zeta_{a}\left(\kappa_{\Sigma}+\zeta_{\Sigma} \partial_{t}\right) \dot{x}-\kappa_{a}\left(\kappa_{\Sigma}+\zeta_{\Sigma} \partial_{t}\right) x=-\left[\kappa_{1} \kappa_{2}+\left(\zeta_{1} \kappa_{2}+\zeta_{2} \kappa_{1}\right) \partial_{t}+\zeta_{1} \zeta_{2} \partial_{t}^{2}\right] x \\
\kappa_{\Sigma}=\kappa_{1}+\kappa_{2}, \quad \zeta_{\Sigma}=\zeta_{1}+\zeta_{2} .
\end{gathered}
$$

Turning to zero coefficients in front of $x$ and $\ddot{x}$, one sees that force $f_{b}$ satisfies the equations for the Maxwell chain if

$$
\zeta_{a}=\frac{\zeta_{1} \zeta_{2}}{\zeta_{1}+\zeta_{2}}, \quad \kappa_{a}=\frac{\kappa_{1} \kappa_{2}}{\kappa_{1}+\kappa_{2}} .
$$

These conditions correspond to sequential connections of viscous and elastic elements defined by Eq. (2.2). After substituting formulas (2.18) in the coefficient of $\dot{x}$ in Eq. (2) and a simple algebra the equation transforms to

$$
\left(\kappa_{\Sigma}+\zeta_{\Sigma} \partial_{t}\right) f_{b}=-\frac{\left(\zeta_{1} \kappa_{2}-\zeta_{2} \kappa_{1}\right)^{2}}{\zeta_{\Sigma} \kappa_{\Sigma}} \dot{x} .
$$

Then the LODE form for the response force of the Maxwell chain comes out as

$$
f_{b}=A \psi ; \quad\left(1+\tau_{\Sigma} \partial_{t}\right) \psi=-\zeta_{\Sigma} \dot{x}, \quad A=\left(\frac{\zeta_{1} \kappa_{2}-\zeta_{2} \kappa_{1}}{\zeta_{\Sigma} \kappa_{\Sigma}}\right)^{2}, \quad \tau_{\Sigma}=\zeta_{\Sigma} / \kappa_{\Sigma} .
$$

Therefore, a sequential connection of two Kelvin chains is equivalent to a scheme comprising a renormalized Kelvin and Maxwell chains connected in parallel. It is worth a note that the partial contribution of the Maxwell part to the resistance force depends on the material parameters of the initial scheme. 
Namely, if somehow $\zeta_{1} \kappa_{2}=\zeta_{2} \kappa_{1}$ and, thus, $A=0$ (see (2.20)), the Maxwell contribution falls out from the equivalent scheme.

In Ref. [26] to model the rheology of amebae cytosol, a simple scheme comprising a viscous element and a Kelvin chain connected sequentially was used. In rheophysics, this combination is known as Jeffreys viscoelastic fluid [17]. Let us construct an equivalent scheme for this medium $[27,28]$. The initial model (see Fig. 4) is described by equations

$$
\left\{\begin{array}{l}
x=x_{1}+x_{2}, \quad f=f_{1}+f_{2} \\
f_{1}=-\zeta_{1} \dot{x}_{1}, \quad f_{2}=-\kappa_{2} \hat{R}_{2} x_{2} .
\end{array}\right.
$$
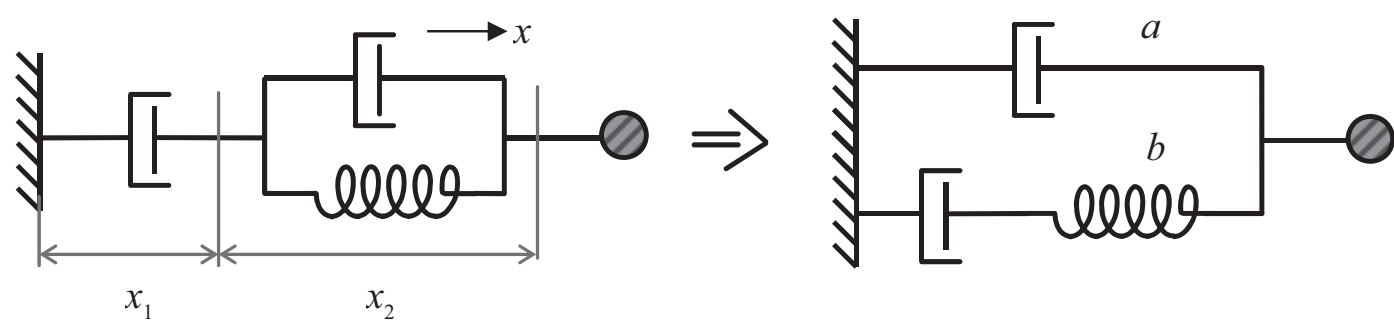

FigurE 4. Jeffreys rheological scheme (left) and its equivalent LODE representation (right). force

Eliminating from Eqs. (2.21) displacements $x_{1}$ and $x_{2}$ as it is described in above, one gets the response

$$
\left(1+\frac{\zeta_{1}+\zeta_{2}}{\kappa_{2}} \partial_{t}\right) f=-\zeta_{1}\left(1+\frac{\zeta_{2}}{\kappa_{2}} \partial_{t}\right) \dot{x}
$$

For the equivalent LODE scheme (Fig. 4, right) the response force is given by equations

$$
\left\{\begin{array}{l}
f=-\zeta_{a} \dot{x}+f_{b} \\
\left(1+\tau_{b} \partial_{t}\right) f_{b}=-\zeta_{b} \dot{x}
\end{array}\right.
$$

Substituting the first of them in (2.22), one obtains equation for the force $f_{b}$, which assumes the Maxwell form if the coefficient of $\ddot{x}$ turns to zero. From this condition follows the expression for the effective viscosity of two sequential viscous elements (Fig. 4):

$$
\zeta_{a}=\frac{\zeta_{1} \zeta_{2}}{\zeta_{1}+\zeta_{2}}
$$

Comparison of the obtained equation for $f_{b}$ with (2.22) renders the other parameters of the equivalent LODE model:

$$
\zeta_{b}=\frac{\zeta_{1}^{2}}{\zeta_{1}+\zeta_{2}}, \quad \kappa_{b}=\kappa_{2}\left(\frac{\zeta_{1}}{\zeta_{1}+\zeta_{2}}\right)^{2}
$$

Probably, the most non-trivial scheme is the sequence of Maxwell and Kelvin chains, Fig. 5, left. From its topology, one may surmise that the equivalent model would consist of two Maxwell chains working in parallel, Fig. 5, right.

Analyzing the initial pattern, one sees that it is described by the set

$$
\left\{\begin{array}{l}
x=x_{1}+x_{2}, f_{1}=f_{2}=f \\
\hat{R}_{1} f_{1}=-\zeta_{1} \dot{x}_{1}, f_{2}=-\kappa_{2} \hat{R}_{2} x_{2} .
\end{array}\right.
$$




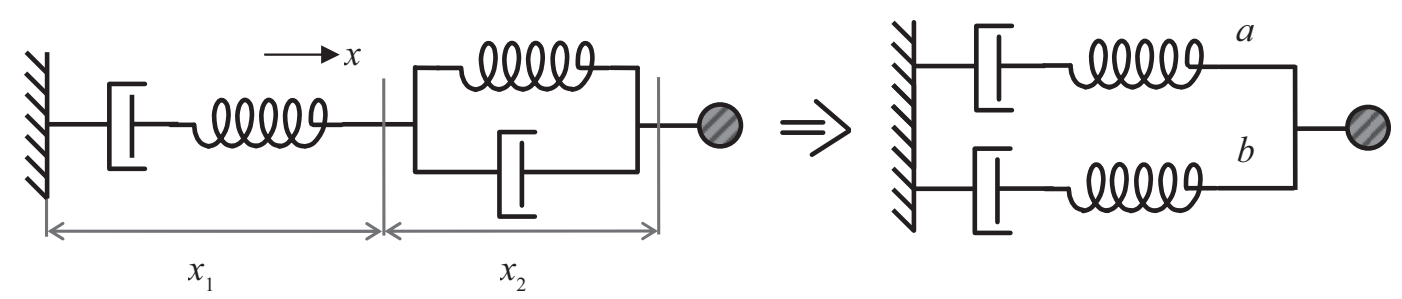

Figure 5. Sequence of Maxwell and Kelvin chains (left), their equivalent LODE scheme (right).

Upon eliminating from it the "non-observable" variables $x_{1}$ and $x_{2}$, the equation for the response force takes the form

$$
\left[1+\left(\tau_{1}+\tau_{2}+\frac{\zeta_{1}}{\kappa_{2}}\right) \partial_{t}+\tau_{1} \tau_{2} \partial_{t}^{2}\right] f=-\zeta_{1}\left(1+\tau_{2} \partial_{t}\right) \dot{x}
$$

To find the parameters of the LODE model, we write the equations determining the response force of the equivalent scheme of Fig. 5, right:

$$
f=f_{a}+f_{b}, \quad \hat{R}_{a} f_{a}=-\zeta_{a} \dot{x}, \quad \hat{R}_{b} f_{b}=-\zeta_{b} \dot{x} .
$$

The sought for equation is

$$
\left[1+\left(\tau_{a}+\tau_{b}\right) \partial_{t}+\tau_{a} \tau_{b} \partial_{t}^{2}\right] f=-\left[\zeta_{a}+\zeta_{b}+\zeta_{a} \zeta_{b}\left(\frac{1}{\kappa_{a}}+\frac{1}{\kappa_{b}}\right) \partial_{t}\right] \dot{x}
$$

Comparison of the equations for the initial and equivalent patterns connects the material parameters of both schemes:

$$
\begin{gathered}
\tau_{a}+\tau_{b}=\tau_{1}+\tau_{2}+\frac{\zeta_{1}}{\kappa_{2}}, \quad \tau_{a} \tau_{b}=\tau_{1} \tau_{2}, \\
\zeta_{a}+\zeta_{b}=\zeta_{1}, \quad \zeta_{a} \zeta_{b}\left(\frac{1}{\kappa_{a}}+\frac{1}{\kappa_{b}}\right)=\frac{\zeta_{1} \zeta_{2}}{\kappa_{2}} .
\end{gathered}
$$

The solution of this set gives

$$
\zeta_{a}+\zeta_{b}=\zeta_{1}, \quad \kappa_{a}+\kappa_{b}=\kappa_{1},
$$

and yields the equation for the retardation time:

$$
\tau^{2}-\left(\tau_{1}+\tau_{2}+\frac{\zeta_{1}}{\kappa_{2}}\right) \tau+\tau_{1} \tau_{2}=0
$$

From the latter one gets

$$
\tau_{a, b}=\frac{1}{2}\left(\tau_{1}+\tau_{2}+\frac{\zeta_{1}}{\kappa_{2}} \pm \sqrt{\left(\tau_{1}+\tau_{2}+\frac{\zeta_{1}}{\kappa_{2}}\right)^{2}-4 \tau_{1} \tau_{2}}\right),
$$

and after some transformations (with allowance for Eqs. (2.31) arrives at the expressions for the equivalent viscosity and elasticity coefficients:

$$
\left\{\begin{array}{l}
\zeta_{a}=\frac{\zeta_{1}}{2}\left(1+\frac{\tau_{1}-\tau_{2}+\zeta_{1} / \kappa_{2}}{\sqrt{\left(\tau_{1}+\tau_{2}+\zeta_{1} / \kappa_{2}\right)^{2}-4 \tau_{1} \tau_{2}}}\right), \zeta_{b}=\zeta_{1}-\zeta_{a} \\
\kappa_{a}=\frac{\kappa_{1}}{2}\left(1+\frac{\tau_{1}-\tau_{2}-\zeta_{1} / \kappa_{2}}{\sqrt{\left(\tau_{1}+\tau_{2}+\zeta_{1} / \kappa_{2}\right)^{2}-4 \tau_{1} \tau_{2}}}\right), \kappa_{b}=\kappa_{1}-\kappa_{a} .
\end{array}\right.
$$


It is worth noting that for the schemes of Fig. 5 does not hold none of the seemingly obvious relations between the parameters of the initial and equivalent schemes, viz. $\zeta_{a}^{-1}=\zeta_{1}^{-1}+\zeta_{2}^{-1}$ and $\kappa_{b}^{-1}=\kappa_{1}^{-1}+\kappa_{2}^{-1}$.

The above-presented examples infer an important conclusion. A rheological scheme of arbitrary complexity may be reduced to an equivalent one, the most general form of which is given by the set

$$
\begin{aligned}
& \left\{\begin{array}{l}
f=-\zeta \dot{x}-\kappa x+\sum_{n=1}^{N} a_{n} f_{n}, \\
\left(1+\tau_{n} \partial_{t}\right) f_{n}=-\zeta_{n} \dot{x},
\end{array}\right. \\
& \tau_{n}=\zeta_{n} / \kappa_{n}, 0 \leq a_{n} \leq 1
\end{aligned}
$$

as illustrated in Fig. 6. Evidently, this scheme is a parallel set comprising one Kelvin and several Maxwell chains. The analysis proves also that the simplest correct model for a viscoelastic medium of the considered type is the Jeffreys fluid, i.e., the case of Eq. (2.35) with $\kappa=0$ and $a_{1}=1$. The pertinent LODE set of dynamic equations for a particle (save for the stochastic forces) is

$$
\left\{\begin{array}{l}
\dot{x}=v \\
m \dot{v}=-\zeta v+Q-\frac{\partial U}{\partial x}, \\
\left(1+\tau_{M} \partial_{t}\right) Q=-\zeta_{M} Q .
\end{array}\right.
$$

Here, along with the retarded friction force $Q$, an external force $-\partial U / \partial x$ acting on the particle is introduced.

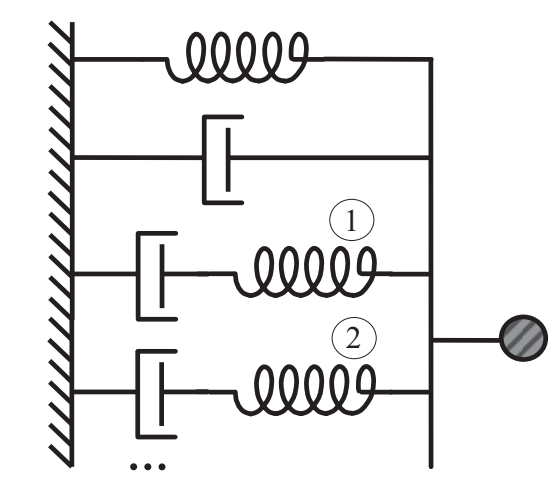

Figure 6. General rheological scheme.

\section{Equations of the particle motion with allowance for thermal fluctuations}

In the preceding section, the response of a viscoelastic medium to the motion of a solid spherical particle inside it was analyzed. Hereby, we show how one has to correctly add thermal fluctuations to this dynamics, thus passing to the Langevin equations. There exist several ways of introducing "white" thermal noise in the dynamic equations, see [21-24], for example. In our view, the most simple and well justified from the physical viewpoint method had been developed by Klimontovich [23], in below we follow this line.

Using the LODE representation of the response force of the viscoelastic medium, the equation of motion for a particle subjected to fluctuational forces could be presented as a set of stochastic equations

$$
\dot{x}_{i}=A_{i}(x)+y_{i}(t), \quad\left\langle y_{i}(t) y_{j}\left(t^{\prime}\right)\right\rangle=2 D_{i j} \delta\left(t-t^{\prime}\right),
$$


where $x_{i}$ are the components of the phase variables of the system, $\boldsymbol{A}$ the drift vector, $D_{i j}$ the matrix of diffusion coefficients (noise intensities). From the set of Langevin equations (3.1) for random functions $x_{i}(t)$ one may pass to the equation for the distribution function for the phase variables. For that purpose, we introduce the phase density

$$
N(\boldsymbol{x}, t)=\frac{1}{N} \sum_{\alpha=1}^{N} \delta\left(\boldsymbol{x}_{\alpha}-\boldsymbol{x}_{\alpha}(t)\right) ;
$$

here $N$ is the total number of particles in the system. It is defined by integral over the whole phase space:

$$
\int d \boldsymbol{x} N(\boldsymbol{x}, t)=1
$$

and is assumed to be constant.

In the motion processes the number of particles is conserved and, due to that, the phase density obeys the continuity equation

$$
\frac{\partial}{\partial t} N+\frac{\partial}{\partial x_{i}}\left(\dot{x}_{i} N\right)=0
$$

The single-particle distribution function $W(\boldsymbol{x}, t)$ is defined as the probability to find a particle at a given time instant in a given space point; the normalization condition for this function is

$$
\int W(\boldsymbol{x}, t) d \boldsymbol{x}=1 .
$$

The relation between $W(\boldsymbol{x}, t)$ and $N(\boldsymbol{x}, t)$ follows from the fact that any observable physical quantity is evaluated as the ensemble average. Therefore, one has

$$
\langle\psi\rangle=\int d \boldsymbol{x} \psi(\boldsymbol{x}) W(\boldsymbol{x}, t) .
$$

On the other hand, the averaging could be done explicitly:

$$
\langle\widetilde{\psi}\rangle=\frac{1}{N} \sum_{\alpha}^{N} \int_{t}^{t+\tau} \frac{d t^{\prime}}{\tau} \psi\left(x_{\alpha}\left(t^{\prime}\right)\right),
$$

that is to integrate the motion of all the particles of the system over all the possible trajectories and while smoothing them with respect to the time $\tau$. The value of $\tau$ is assumed to be large in comparison with the correlation time of the stochastic forces, and, thus, depends on the nature of the quantity measured. For a vast majority of physical systems, both averaging methods are equivalent (ergodicity): $\langle\psi\rangle=\langle\widetilde{\psi}\rangle$.

Transformation of expression (3.7) with the aid of definition of $\delta$-function, yields

$$
\begin{aligned}
\langle\widetilde{\psi}\rangle=\frac{1}{N} \sum_{\alpha=1}^{N} \int_{t}^{t+\tau} \frac{d t^{\prime}}{\tau} \int d \boldsymbol{x} \psi(\boldsymbol{x}) \delta & \left(\boldsymbol{x}-\boldsymbol{x}_{\alpha}(t)\right) \\
& =\int d \boldsymbol{x} \psi(\boldsymbol{x}) \frac{1}{N} \sum_{\alpha=1}^{N} \int_{t}^{t+\tau} \frac{d t^{\prime}}{\tau} \delta\left(\boldsymbol{x}-\boldsymbol{x}_{\alpha}(t)\right)=\int d \boldsymbol{x} \psi(\boldsymbol{x})\langle N\rangle .
\end{aligned}
$$

Comparison of formulas (3.6) and (3.8) renders the relation

$$
W(\boldsymbol{x}, t)=\langle N(\boldsymbol{x}, t)\rangle,
$$


so that the phase density may be presented as

$$
N(\boldsymbol{x}, t)=W(\boldsymbol{x}, t)+\delta N, \quad \delta N=N-\langle N\rangle .
$$

Substituting expansion (3.10) in the continuity equation (3.4) one gets

$$
\frac{\partial}{\partial t} W(\boldsymbol{x}, t)+\frac{\partial}{\partial x_{i}}\left(A_{i} W(\boldsymbol{x}, t)\right)+\frac{\partial}{\partial x_{i}}\left\langle y_{i}(t) \delta N(t)\right\rangle=0,
$$

where $\left\langle y_{i}\right\rangle=0$ and $\langle\delta N\rangle=0$.

To find the correlation functions of stochastic forces and fluctuations of phase density, we subtract Eq. (3.11) from the continuity equation. This gives

$$
\frac{\partial}{\partial t} \delta N+\frac{\partial}{\partial x_{i}}\left(A_{i} \delta N\right)+\frac{\partial}{\partial x_{i}}\left[y_{i} W+\left(y_{i} \delta N-\left\langle y_{i} \delta N\right\rangle\right)\right]=0 .
$$

In the sought for correlation function it suffices to retain only the fast (singular) part of the solution. In the linear (with respect to fluctuations) approximation one has

$$
\delta N(\boldsymbol{x}, t) \cong-\lim _{\tau \rightarrow 0} \frac{\partial}{\partial x_{j}} \int_{t-\tau}^{t} d t^{\prime}\left(y_{j}\left(t^{\prime}\right) W\left(\boldsymbol{x}, t^{\prime}\right)\right)
$$

we remind that time $\tau$ is far larger than the correlation time of fluctuations. Substitution of solution (3.13) in (3.11) finally renders a closed kinetic equation (Fokker-Planck equation):

$$
\frac{\partial}{\partial t} W(\boldsymbol{x}, t)+\frac{\partial}{\partial x}\left(A_{i} W\right)=\frac{\partial^{2}}{\partial x_{i} \partial x_{j}}\left(D_{i j} W\right)
$$

for the probability density (distribution function) of the system.

Let us apply this theoretical scheme to the central problem of this work: description of Brownian motion in the Jeffreys fluid [27]. In the simplest form (1D translational motion) the pertinent set of equations is

$$
\left\{\begin{array}{l}
\dot{x}=v \\
m \dot{v}=-\zeta_{N} v+Q-\left(\frac{\partial U}{\partial x}\right)+y_{N}(t), \\
\left(1+\tau_{M} \partial_{t}\right) Q=-\zeta_{M} v+y_{M}(t) \\
\left\langle y_{\alpha}(t) y_{\beta}(t+\tau)\right\rangle=2 \delta_{\alpha} \beta D_{\alpha} \delta(t)
\end{array}\right.
$$

where Greek subscripts assume the values: $N$ for a Newtonian and $M$ for the Maxwellian response of the system.

The fluctuation-dissipation theorem [29] determines the relation between the dissipative parameters of the medium and the noise intensity:

$$
D_{\alpha}=T \zeta_{\alpha}, \quad \alpha=M, N
$$

This fundamental result follows from the Fokker-Planck equation. To demonstrate this, we, first, introduce the phase density

$$
N(x, v, Q, t)=\frac{1}{N} \sum_{\alpha=1}^{N} \delta\left(x-x_{\alpha}(t)\right) \delta\left(v-v_{\alpha}(t)\right) \delta\left(Q-Q_{\alpha}(t)\right) .
$$

As long as we neglect the interparticle interactions, the equation of motions for all the particles have the same form, so that the subscript numbering the particles in Eq. (3.15) may be omitted. Then the 
equation for distribution function $W=W(x, v, Q, t)$ writes

$$
\begin{aligned}
\frac{\partial}{\partial t} W+\left[v \frac{\partial}{\partial x}-\frac{1}{m}\left(\frac{\partial U}{\partial x}\right)\right. & \left.\frac{\partial}{\partial v}\right] W+\left(\frac{Q}{m} \frac{\partial}{\partial v}-v \kappa_{M} \frac{\partial}{\partial Q}\right) W \\
& =\frac{\zeta_{N}}{m} \frac{\partial}{\partial v}(v W)+\gamma_{M} \frac{\partial}{\partial Q}(Q W)-\frac{1}{m} \frac{\partial}{\partial v}\left\langle y_{N} \delta N\right\rangle-\gamma_{M} \frac{\partial}{\partial Q}\left\langle y_{M} \delta N\right\rangle,
\end{aligned}
$$

where $\gamma_{M}=1 / \tau_{M}=\kappa_{M} / \zeta_{M}$. Evaluating the correlation functions in (3.18) with the aid of formula for the singular part of the phase density fluctuation

$$
\delta N=-\lim _{\tau \rightarrow 0} \int_{t-\tau}^{t}\left[\frac{1}{m} \frac{\partial}{\partial v} y_{N}\left(t^{\prime}\right)+\gamma_{M} \frac{\partial}{\partial Q} y_{M}\left(t^{\prime}\right)\right] W d t^{\prime},
$$

we arrive at the sought for kinetic equation

$$
\begin{aligned}
\frac{\partial}{\partial t} W+\left[v \frac{\partial}{\partial x}-\frac{1}{m}\left(\frac{\partial U}{\partial x}\right) \frac{\partial}{\partial v}\right] W & +\left(\frac{Q}{m} \frac{\partial}{\partial v}-v \kappa_{M} \frac{\partial}{\partial Q}\right) W \\
& =\gamma_{m} \frac{\partial}{\partial v}\left(v+\frac{D_{N}}{\zeta_{N} m} \frac{\partial}{\partial v}\right) W+\gamma_{M} \frac{\partial}{\partial Q}\left(Q+\gamma_{M} D_{M} \frac{\partial}{\partial Q}\right) W
\end{aligned}
$$

with $\gamma_{m}=1 / \tau_{m}=\zeta_{N} / m$.

In equation (3.20) the noise intensities $D_{N}$ and $D_{M}$ are yet unknown. They are evaluated using the fact that in thermodynamic equilibrium the distribution function should have the Maxwell-Boltzmann form. We note that, along with customary - kinetic and potential - contributions to the particle energy, one has to introduce a pseudo-potential caused by the viscoelasticity of the medium surrounding the particle. From Eq. (3.20) it follows that this pseudo-potential is quadratic with respect to the retarded force $Q$ :

$$
W_{0}(x, v, Q) \sim \exp \left\{-\frac{1}{T}\left[\frac{m v^{2}}{2}+U(x)+\frac{\sigma Q^{2}}{2}\right]\right\} .
$$

On substituting $W_{0}$ in the Fokker-Planck equation (3.20), the first drift term disappears identically. The condition, which turns to zero the second drift term, defines the pseudo-potential parameter:

$$
\sigma=1 / \kappa_{M}
$$

thus yielding the equilibrium distribution in the form

$$
W_{0}(x, v, Q) \propto \exp \left\{-\frac{1}{T}\left[\frac{m v^{2}}{2}+U(x)+\frac{Q^{2}}{2 \kappa_{M}}\right]\right\} .
$$

This defines the additional term that has first appeared in (3.21). It is the direct consequence of the viscoelasticity. Each displacement $\delta x$ of the particle during the time $\delta t \ll \tau_{M}$, according to Eq. (3.15), generates the restoring force

$$
Q=-\kappa_{M} \delta x
$$

to which one should ascribe the harmonic potential $\frac{1}{2} \kappa_{M}(\delta x)^{2}$. Rewriting it in terms of the response force $Q$ one gets the additional term in the Maxwell-Boltzmann distribution (3.23). In other words, on undergoing Brownian dynamics, the particle induces in a viscoelastic medium a certain amount of elastic energy.

Finally, the equilibrium solution of Eq. (3.20) requires that the right-hand should turn to zero. For that, on the phase variables $v$ and $Q$ the so-called potentiality conditions should be imposed:

$$
\left(v+\frac{D_{N}}{m \zeta_{N}} \frac{\partial}{\partial v}\right) W_{0}=0, \quad\left(Q+\frac{\kappa_{M} D_{M}}{\zeta_{M}} \frac{\partial}{\partial Q}\right) W_{0}=0
$$

These equations prove the fluctuation-dissipation theorem (3.15). 


\section{Translational Brownian motion in Jeffreys fluid}

Mean square displacement (MSD) is a fundamental characteristic of Brownian motion. This quantity is well measurable with a number of methods [1]. In this section, we consider evaluation of MSD in Jeffreys fluid, first discussed in our work [27]. We begin with the non-inertial limit $m \rightarrow 0$, which is valid at the time intervals $t \gg \tau_{m}=m / \zeta_{N}$. For a spherical particle of radius $a$ the estimate yields

$$
\tau_{m}=\frac{m}{\zeta_{N}}=\frac{4 \pi}{3} \frac{\rho a^{3}}{6 \pi \eta_{N} a} \simeq 10^{-6}\left[\frac{a^{2}\left(\mu \mathrm{m}^{2}\right)}{\eta(\mathrm{sP})}\right] \mathrm{s} .
$$

As seen, for the reference particle size $a \sim 1 \mu \mathrm{m}$ and the Newtonian viscosity $\eta \sim 1 \mathrm{P}$, the value of $\tau_{m}$ is about $10^{-6} \mathrm{~s}$. This means that inertial effects matter only at the frequencies $f \geq 10 \mathrm{MHz}$.

In the non-inertial limit the number of phase variables reduces, and the set of Langevin equations (3.15) transforms to ${ }^{1}$

$$
\left\{\begin{array}{l}
\dot{x}=\frac{1}{\zeta_{N}}\left[Q+y_{N}(t)\right], \\
\dot{Q}=-\widetilde{\gamma} Q+\kappa_{M}\left[\frac{y_{M}(t)}{\zeta_{M}}-\frac{y_{N}(t)}{\zeta_{N}}\right],
\end{array}\right.
$$

where $\widetilde{\gamma} \equiv \gamma_{M}+\gamma_{N}$ and $\gamma_{N}=\kappa_{M} / \zeta_{N}$. In an isotropic medium, the particle displacements are equiprobable in any direction, so that $\langle x\rangle_{t}=0$. The equation for MSD $\left\langle x^{2}\right\rangle$ is obtained from the first of equations (4.2) and has the form

$$
\frac{\partial}{\partial t}\left\langle x^{2}\right\rangle=\frac{2}{\zeta_{N}}\left[\langle x Q\rangle+\left\langle x y_{N}\right\rangle\right]
$$

According to the same basic equation, the instantaneous correlation function obeys the relation

$$
\left(\frac{\partial}{\partial t}+\widetilde{\gamma}\right)\langle x Q\rangle=\kappa_{M}\left\langle x\left(\frac{y_{M}}{\zeta_{M}}-\frac{y_{N}}{\zeta_{N}}\right)\right\rangle+\frac{1}{\zeta_{N}}\left[\left\langle Q^{2}\right\rangle+T\left\langle y_{N} Q\right\rangle\right] .
$$

The fast singular part of the displacement follows directly from the first of equations (4.2):

$$
\delta x=\frac{1}{\zeta_{N}} \lim _{\tau \rightarrow 0} \int_{t-\tau}^{t} d t^{\prime} y_{N}\left(t^{\prime}\right) \equiv \hat{I}\left(y_{N}^{\prime} / \zeta_{N}\right), \quad \delta Q=\kappa_{M} \hat{I}\left(\frac{y_{M}^{\prime}}{\zeta_{M}}-\frac{y_{N}^{\prime}}{\zeta_{N}}\right)
$$

where integration operator $\hat{I}$ over an infinitesimal time interval preceding the present instant is introduced. Substitution of (4.5) in the correlation functions in the moment equations (4.3) and (4.4) under assumption of statistical equilibrium, transforms them to

$$
\frac{\partial}{\partial t}\left\langle x^{2}\right\rangle=\frac{2}{\zeta_{N}}\langle x Q\rangle+2 \frac{T}{\zeta_{N}}, \quad\left(\frac{\partial}{\partial t}+\widetilde{\gamma}\right)\langle x Q\rangle=-\frac{\kappa_{M} T}{\zeta_{N}}
$$

As the initial position of the particle is defined by $x(t=0)=0$, the initial conditions for the set (4.6) are homogeneous:

$$
\left\langle x^{2}\right\rangle_{t=0}=0, \quad\langle x Q\rangle_{t=0}=0 .
$$

Solving Eqs. (4.6) by standard method, we write the result for 3D displacement $\Delta \boldsymbol{r}$ in order to facilitate comparison with experiment. For an isotropic medium one has [28]:

$$
\left\langle(\Delta \boldsymbol{r})^{2}\right\rangle=\frac{6 T}{\zeta_{N}(1+q)}\left\{t+\frac{q \tau_{M}}{1+q}\left[1-e^{-\frac{(1+q) t}{\tau_{M}}}\right]\right\}, \quad q \equiv \frac{\zeta_{M}}{\zeta_{N}}=\frac{\eta_{M}}{\eta_{N}} .
$$

\footnotetext{
${ }^{1}$ We consider here free Brownian motion setting $U=0$.
} 
This expression has a clear physical meaning. In the considered model, friction is due to two mechanisms. One of them has zero response time and yields fast $(q \rightarrow 0)$ diffusion with low Newtonian viscosity $\eta_{N}$. The response of the other mode is retarded by the Maxwell time $\tau_{M}$ and, thus, causes slow $(q \rightarrow \infty)$ diffusion with high viscosity $\eta_{M}$. On the time intervals shorter than $\tau_{M}$, the Maxwell mechanism works as the restoring force, thus inducing dynamic localization of the particle. If the time resolution of the observation is sufficiently high, then both types of relaxation are observable together with their crossover effect, which manifests itself as a virtual (dynamic) localization of the particle.

Consider this model in the limit $q \gg 1$, which, as it would be shown below, is the case for micellar solutions and polymer colloids. At short times, $\widetilde{\gamma} t \ll 1$, formula (4.8) reduces to the relation

$$
\left\langle(\Delta \boldsymbol{r})^{2}\right\rangle \simeq \frac{6 T}{\zeta_{N}} t\left(1-\frac{t}{2 \tau_{N}}\right), \quad \tau_{N}=\frac{\tau_{M}}{q}=\frac{\zeta_{N}}{\kappa_{M}},
$$

that describes the fast diffusion regime. In the intermediate time interval $\tau_{N} \ll t \ll \tau_{M}$, the dynamic localization (confinement) of the particle takes place:

$$
\left\langle(\Delta r)^{2}\right\rangle \simeq 6 T / \kappa_{M}
$$

since here the Maxwell chain responds as an elastic element. At long times $\left(t \gg \tau_{M}\right)$ the Maxwell viscosity adds to relaxation, so that the slow diffusion regime is established, where both viscous elements of the Jeffreys scheme work. For this mode, formula (4.8) yields

$$
\left\langle(\Delta \boldsymbol{r})^{2}\right\rangle \simeq \frac{6 T}{\zeta_{N}(1+q)}\left[t+\frac{q \tau_{M}}{1+q}\right], \quad t>\frac{\tau_{M}}{1+q},
$$

Comparison of expressions (4.11) and (4.9) shows that the long-time diffusion is $(1+q)$ times slower than the short-time one. The transition (crossover) from fast to slow diffusion takes place at the time interval $\tau_{N}<t<q \tau_{N}$, during which the particle MSD virtually does not change, i.e., the particle is dynamically localized (confined). Note that at $q \gg 1$ (fluids with strong viscoelasticity) the duration of this event is much larger than $\tau_{N}$.

All the above-described peculiarities of MSD were observed in Ref. [30]. There the microrheological probing of water surfactant solution: a mixture of cetyltrimethylammonium bromide and potassium bromide (CTAB-KBr). Under appropriate concentrations this system produces worm-like micelles (length $\sim 1 \mu \mathrm{m}$, diameter $\sim 5 \mathrm{~nm}$ ), which are very flexible (persistent length $\sim 20 \mathrm{~nm}$ ). At the same time, some amount of the surfactant molecules are present in the solution in non-bounded state. Between both components, thermodynamic equilibrium is established so that the molecular contour length is a variable parameter. The systems of such type are known as living polymers.

In Fig. 7a taken from [27], the points show the MSD data of Ref. [30] obtained with spherical probing particles of radius $1 \mu \mathrm{m}$ in the solution $[\mathrm{CTAB}]=1 \mathrm{~g} / \mathrm{cm}^{3}$ and $[\mathrm{KBr}]=2 \mathrm{M}$ at $36 \mathrm{C}$. As seen, MSD grows linearly at short times (fast diffusion), then makes a plateau, and resumes the linear growth in the slow diffusion regime. If the micellar solution CTAB-KBr to be sufficiently homogeneous on the scale of the probe size, then application of formula (4.8) is completely justified. The Maxwell time $\tau_{M}=1.05 \mathrm{~s}$ is given in [30], the nondimensional coefficient $q$ of the model is determined by the inflexion of the curve (4.8) when turning to the plateau. In experiment, this behavior of MSD was observed with enough accuracy. Comparison with the model gave $q=1.1 \times 10^{4}$, thus confirming the limit $q \gg 1$ that we use. Note that, according to the definition of parameter $q$, it is the ratio of the viscosities $\eta_{M} / \eta_{N}$. Therefore, in this colloid the Newtonian viscosity is four orders lower than the Maxwell one, and is about $0.8 \mathrm{P}$. The last of the parameters of formula (4.8) may be written as $\zeta_{N}=6 \pi a \eta_{M} / q$. Substituting there the found value of $q$ and the viscosities $\eta_{M}=8.8 \times 10^{3} \mathrm{P}$ and $a=0.48 \mu \mathrm{m}$ given in [30], we get $\zeta_{N}=7.2 \times 10^{-4} \mathrm{~g} / \mathrm{s}$.

As seen, formula (4.8) enables one to fairy well fit the measurement results in a rather wide (more than six decades) range. This interval covers both diffusion regimes as well as their crossover. However, 

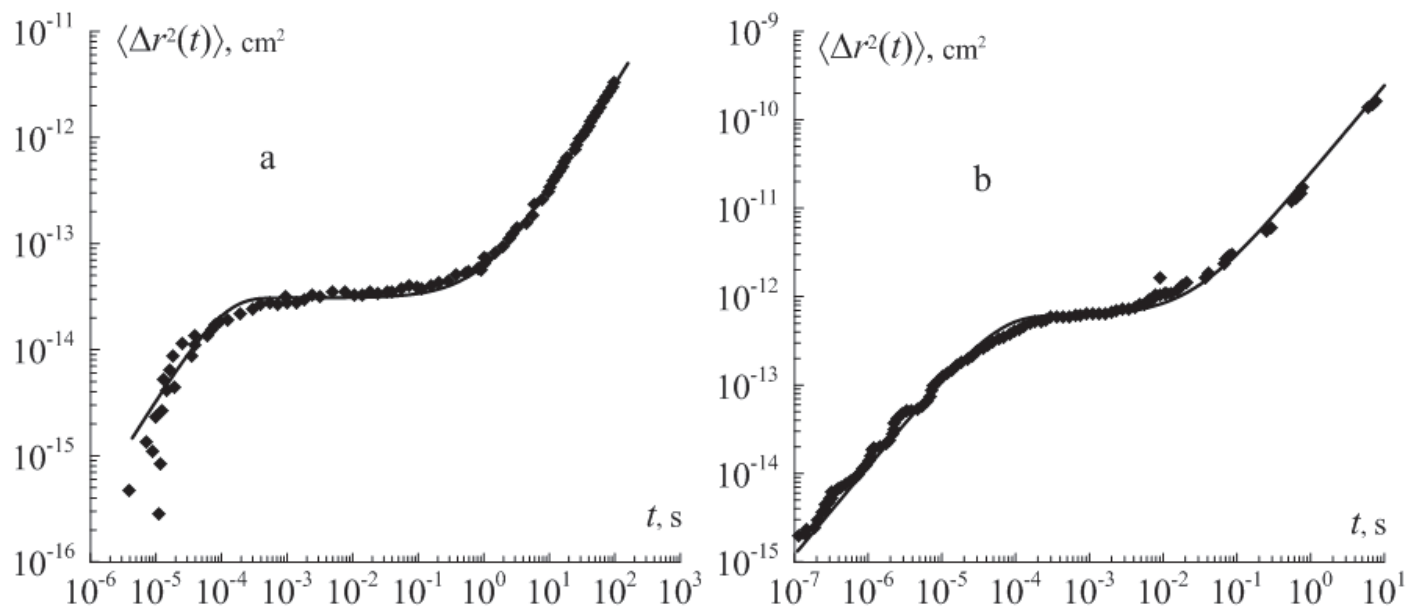

FiguRE 7. Application of formula (4.11) to diffusion measurements with: (a) particles of diameter $1 \mu \mathrm{m}$ in micellar CTAB-KBr [30] solution with parameters $\tau_{M}=1.05 \mathrm{~s}$ and $q=\tau_{M} / \tau_{N} \simeq 1.1 \times 10^{4}$; (b) particles of diameter $0.7 \mu \mathrm{m}$ in giant-micelle solution [31] with parameters $\tau_{M}=0.08 \mathrm{~s}$ and $q=690$.

the theory and experiment deviate at very short times $\left(t<10^{-5} \mathrm{~s}\right)$. One of the possible causes is the reduced accuracy of measurement, note the enhanced spread of the experimental points in this region.

Fig. 7b, first presented in Ref. [28], gives another example of applying Eq. (4.8) to measurement data. The tested system is a solution of hexa-ethylene glycol mono-n-hexadecyl ether that forms giant micelles [31]. The ratios $\tau_{M}=\eta_{M} / G$ and $T / a G$ given in [31] along with the MSD data facilitate fitting as they enable one to estimate $q$, see the figure legend. Once again, Eq. (4.8) proves to be well capable of describing the results. Numerous examples of the same type are given in Refs. [32,33].

\section{Brownian motion in Jeffreys fluid with allowance for the particle inertia}

A detailed study of this problem is given in Ref. [28]. Here we remark only its essential points. It turned out that calculation of MSD is most facile when using the velocity autocorrelation function. For a stationary process this function is symmetrical with respect to the difference of its arguments, so the sought for expression could be presented in the form

$$
\left\langle x^{2}\right\rangle=2 \int_{0}^{t} d t^{\prime} \int_{0}^{t^{\prime}} d t^{\prime \prime}\left\langle v v_{t^{\prime}-t^{\prime \prime}}\right\rangle .
$$

Hereafter for brevity we denote $\left\langle v v_{t}\right\rangle \equiv\langle v(0) v(t)\rangle$ counting the displacement from the initial position $x(t=0)$. For a free particle $U=0$, the set of Langevin equation transforms to the following equations for the correlation functions:

$$
\begin{aligned}
& \left(\frac{\partial}{\partial t}+\gamma_{m}\right)\left\langle v v_{t}\right\rangle=\frac{1}{m}\left\langle v Q_{t}\right\rangle+\frac{1}{m}\left\langle v y_{N}(t)\right\rangle, \\
& \left(\frac{\partial}{\partial t}+\gamma_{M}\right)\left\langle v Q_{t}\right\rangle=-\kappa_{M}\left\langle v v_{t}\right\rangle+\gamma_{M}\left\langle v y_{M}(t)\right\rangle, \quad t>0 ;
\end{aligned}
$$

here $\gamma_{m}=1 / \tau_{m}=\zeta_{N} / m$ and $\gamma_{M}=1 / \tau_{M}=\kappa_{M} / \zeta_{M}$. The cross-correlators of velocity with the random forces present in the right-hand parts, are identical zeroes due to the causality principle. According to it, the state of the system, for example $v(0)$, at a given instant of time does not depend of the action on it 
which would be exerted in future, i.e., of $y(t), t>0$. The initial conditions for this set are the equilibrium values determined by distribution (3.21):

$$
\langle v v\rangle_{t=0}=\left\langle v^{2}\right\rangle=T / m, \quad \frac{\partial}{\partial t}\left\langle v v_{t}\right\rangle_{t=0}=-\gamma_{m}\left\langle v^{2}\right\rangle .
$$

Eliminating cross-correlator $\langle v Q\rangle_{t}$ from the set (5.2), one arrives at the equation for the velocity autocorrelation function:

$$
\left[\left(\frac{\partial}{\partial t}+\gamma_{m}\right)\left(\frac{\partial}{\partial t}+\gamma_{M}\right)+\frac{\kappa_{M}}{m}\right]\left\langle v v_{t}\right\rangle=0
$$

The solution satisfying initial condition (5.3) is

$$
\left\langle v v_{t}\right\rangle=\frac{\left\langle v^{2}\right\rangle}{\lambda_{2}-\lambda_{1}}\left[\left(\lambda_{2}+\gamma_{M}\right) e^{\lambda_{2} t}-\left(\lambda_{1}+\gamma_{M}\right) e^{\lambda_{1} t}\right]
$$

where decrements $\lambda$ are the roots of characteristic equation

$$
\lambda^{2}+\left(\gamma_{m}+\gamma_{M}\right) \lambda+\gamma_{m} \gamma_{M}+\frac{\kappa_{M}}{m}=0
$$

Let us introduce nondimensional material parameter

$$
M \equiv \frac{\tau_{M}}{m} \zeta_{M}=\frac{\zeta_{M}^{2}}{\kappa_{M} m}
$$

that characterizes the relation between inertial, viscous and elasticity forces. For the Maxwell model, the number $M$ (Maxwell number) is the only nondimensional criterion [34]. In the Jeffreys model, the number $M$ pertains exclusively to its Maxwell part, while the ratio of the two viscous elements of the scheme is given by parameter $q$ defined in (4.8). In these notations, the roots of characteristic equation take the form

$$
\lambda_{1,2}=-\frac{1}{2} \gamma_{M}\left[1+\frac{M}{q} \pm \sqrt{\left(1-\frac{M}{q}\right)^{2}-4 M}\right] .
$$

Substitution of the velocity autocorrelation function (5.5) in equation (5.1) after integration yields MSD as

$$
\left\langle x^{2}\right\rangle=\frac{2\left\langle v^{2}\right\rangle}{\lambda_{2}-\lambda_{1}}\left[\frac{\lambda_{2}+\gamma_{M}}{\lambda_{2}}\left(\frac{e^{\lambda_{2} t}-1}{\lambda_{2}}-t\right)-\frac{\lambda_{1}+\gamma_{M}}{\lambda_{1}}\left(\frac{e^{\lambda_{1} t}-1}{\lambda_{1}}-t\right)\right] .
$$

As seen, in the limiting case $t \ll \tau_{m}(\lambda t \ll 1)$ the particle motion is governed mostly by its inertia (ballistic regime): $\left\langle v v_{t}\right\rangle \simeq\left\langle v^{2}\right\rangle\left(1-\gamma_{m} t\right)$. The corresponding reduction of expression (5.9) gives for MSD

$$
\left\langle x^{2}\right\rangle \simeq\left\langle v^{2}\right\rangle t^{2}\left(1-\frac{t}{3 \tau_{m}}\right)
$$

We remark, however, that, as the estimate (4.1) shows, the ballistic (non-Brownian) regime $\left\langle x^{2}\right\rangle \propto t^{2}$ of the particle motion might be resolved only in experiments with very short observation time $\left(t<\tau_{m}\right)$. At long time intervals $\left(t>\tau_{m}\right)$, which is the case for majority of experiments, the above-mentioned noninertial $(m \rightarrow 0)$ regime takes place. Extension of formula (5.9) for 3D case is obvious: $\left\langle(\Delta \boldsymbol{r})^{2}\right\rangle=3\left\langle x^{2}\right\rangle$, thus leading to Eq. (4.8).

Upon certain simple algebraic re-arrangements, one obtains a useful formula presenting the general MSD expression (5.9) in terms of the material parameters of the Jeffreys model:

$$
\left\langle(\Delta \boldsymbol{r})^{2}\right\rangle=\frac{6 T}{\zeta_{N}(1+q)}\left\{t+\tau_{M} \frac{q(M-1)}{(1+q) M}\left[1-\exp \left(-\frac{q+M}{2 \tau_{M} q} t\right) \frac{\operatorname{sh}(\beta t+\psi)}{\operatorname{sh} \psi}\right]\right\} .
$$


Here $2 \beta=\gamma_{M} \sqrt{(M / q-1)^{2}-4 M}$, while the "phase shift" is given by the relation

$$
\operatorname{cth} \psi=\frac{3 M-1+\frac{M(M+1)}{q}}{2 \beta \tau_{M}(M-1)} .
$$

An important conclusion, which follows from considering the motion with allowance for the particle inertia, is the failure of Maxwell model to describe Brownian motion practically in all the time interval, where viscoelasticity is important, i.e., at $t \ll \tau_{M}$. The more so this circumstance should be emphasized that in the literature there exists a number of works, see [35-39], for example, which employ this very approach. Two issues seemingly justify it. For one thing, the Maxwell model is simpler than the Jeffreys model. Second, the Maxwell model is successfully used and does not cause any artifacts in macroscopic rheology. However, when addressing Brownian motion, the situation is different: it turns up that Maxwell scheme displays an essential non-avoidable flaw. Its origin is simple: at the times shorter than $\tau_{M}$ the Maxwell fluid exerts no friction on the particle. As a result, when used to resemble the Brownian particle environment, the Maxwell model predicts non-physical high-quality oscillations, which are never observed.

To clarify this statement, we derive from Eq. (5.8) the conditions, under which a Brownian particle embedded in a Jeffreys fluid might oscillate. According to those formulas, the equation for the neutral curve $(\operatorname{Im} \lambda)$ on the plane of nondimensional parameters (5.7) takes the form

$$
\sqrt{(1-M / q)^{2}-4 M}=0
$$

Considering its roots, one sees that oscillations occur in either of two cases:

$$
\left\{\begin{array}{l}
\text { for } M<\frac{1}{4} \text { at } \frac{M}{1+2 \sqrt{M}}<q<\frac{M}{1-2 \sqrt{M}}, \\
\text { for } M>\frac{1}{4} \text { at } \frac{M}{1+2 \sqrt{M}}<q .
\end{array}\right.
$$

This is illustrated by Fig. 8 first published in Ref. [28], where the oscillatory regime corresponds to the "sector" in between the two neutral curves.

Upon limiting transition to the Maxwell fluid $(q \rightarrow \infty)$ Eqs. (5.13) yield a single border: $M=\frac{1}{4}$. Rearranging it to dimensional form, one has

$$
4 \zeta_{M}^{2}>m K_{M} \quad \Rightarrow \quad \eta_{M}^{2}>m G /(24 \pi a)
$$

where from it follows that the oscillatory motion of the particle emerges when the retarded viscosity dominates over inertia and elasticity. In other words, according to Eq. (5.14) oscillatory relaxation in a Maxwell fluid is the more intense the greater friction. Apparently, this inference contradicts the physical meaning of friction in mechanics. Thus, it has just very formal meaning. Analyzing the situation, one understands that the non-physical conclusion follows from the fact that the Maxwell model lacks the fast (non-retarded) viscosity. Because of that, in the Maxwell fluid any high-frequency excitation of the particle becomes oscillatory. Moreover, the quality factor of this process $Q=2 \sqrt{M}=2 \zeta_{M} / \sqrt{m K_{M}}$ the greater the larger the slow viscosity and the smaller the particle mass. This infers that, upon tending the mass to zero, i.e., in the non-inertial limit, Brownian motion should be oscillatory in any (!) Maxwell fluid. The inanity of this conclusion, in our view, provides an exhaustive ad absurdum proof of impossibility to use the Maxwell model for studies of Brownian motion in viscoelastic media. In this connection, we would like to point out the works [35-39], where oscillations of Brownian particles were predicted on in the framework of Maxwell approximation.

The fundamental advantage of Jeffreys model is that, when applied to the Brownian motion in nonNewtonian fluids, it "regularizes" the theory, and enables one to obtain the results, which are free of 




FiguRE 8. Regions of oscillatory (white) and monotonic (gray) relaxations in the plane of viscoelasticity parameters separated by the branches of the neutral curve; dashes mark the line $M=\frac{1}{4}$; in a Maxwell fluid the region $M>\frac{1}{4}$ corresponds to the oscillatory diffusion whatever $q$.

artifacts. For illustration, we consider a typical viscoelastic system, where $M, q \gg 1$. According to Eq. (5.13), the onset of oscillatory motion takes place on attaining the condition $q>\frac{1}{2} \sqrt{M}$ or, in dimensional form, $\sqrt{m K_{M}}>\zeta_{N} / 2$. Using the definitions of material parameters and substituting in (5.14) the reference values $\tau_{M} \approx 1 \mathrm{~s}, \eta_{N} \sim 1 \mathrm{P}$ and $\rho \approx 1 \mathrm{~g} / \mathrm{cm}^{3}$, we arrive at the estimate

$$
a_{*} \sim \sqrt{\tau_{M} \eta_{N} / 2 q \rho} \sim 1 \mathrm{~cm} / \sqrt{q} .
$$

which shows that in the Jeffreys model oscillations are possible only at $a>a_{*}$. However, as seen from Eq. (5.15), the obtained value of the particle size is in the region, where the particle Brownian motion is entirely negligible. Due to that, the here developed theory, which is based on the Jeffreys model, comes out free of non-physical conclusions and retains its robustness for the simplest rheological scheme (two viscosities) as well as for any of its modification with an arbitrary number of viscosity coefficients.

\section{Mean-square displacement in the particle rotary motion}

Here we consider the in-plane rotation of a particle, that is its rotation about a fixed axis. Assuming that, we simplify the mathematical statement of the problem but nevertheless keep all the relevant physical parameters involved in the description. Due to that, the would-be solution should reveal all the qualitative effects inherent to the system under study. The practice of such simplification (see [24], for example) evidences that its difference from a more complicated case of 3D rotation consists in nondimensional factors of the order of unity.

Let the particle have a permanent dipole moment $\boldsymbol{\mu}$, which lies in the plane of rotation and is "frozen" in the particle body. Consider, first the free Brownian rotation, i.e., the case of zero external field. The orientational state of the particle is described in full by the angle $\theta$, which vector $\boldsymbol{\mu}$ makes with a given coordinate axis. We replace the rheological elements of the Jeffreys scheme by their analogues, which correspond to rotary motion of a spherical particle of radius $a$ :

$$
\kappa_{M}=6 G_{M} V, \quad \zeta_{\alpha}=6 \eta_{\alpha} V, \quad V=4 \pi a^{3} / 3, \quad \alpha=N, M .
$$

The equation of motion for the angular variable $\theta$ follows from Eq. (3.23) upon obvious replacement $x \rightarrow \theta, m \rightarrow I$, where $I$ is the moment of inertia of the particle. Then Eq. (4.8) transforms to the 
expression for the mean-square fluctuation of the phase:

$$
\left\langle(\Delta \theta)^{2}\right\rangle=\frac{2 T}{\zeta_{N}(1+q)}\left\{t+\frac{q}{\widetilde{\gamma}}\left(1-e^{-\widetilde{\gamma} t}\right)\right\}, \quad \widetilde{\gamma}=\frac{1+q}{\tau_{M}}=\gamma_{M}(1+q)
$$

We remind that in the Jeffreys model the friction is generated by two mechanisms connected in parallel, see Fig. 4. The reference response time of Newtonian viscosity is $\tau_{N}=\zeta_{N} / \kappa_{M}$, while the response time of the Maxwell element is $\tau_{M}=\zeta_{M} / \kappa_{M}$. The ratio of these times is rendered by parameter $q=\tau_{M} / \tau_{N}=\zeta_{M} / \zeta_{M}$ characterizing the effect of viscoelasticity of the medium on the moving particle. The case of weak viscoelasticity $(q \ll 1)$ is of no interest because it but slightly differs from the wellstudied case of Brownian motion in a Newtonian fluid [24]. The most important is the case of developed viscoelasticity $(q \gg 1)$, when the reference times of the two viscous elements are substantially different: $\tau_{N} \ll \tau_{M}=q \tau_{N}$. Here the switching between the two types (modes) of diffusion entails a notable effect. From Eq. (6.2) in the short-time limit $t \ll \tau_{N}$ one gets the fast orientational diffusion

$$
\left\langle(\Delta \theta)^{2}\right\rangle \simeq \frac{2 T}{\zeta_{N}} t\left(1-\frac{t}{2 \tau_{N}}\right), \quad t \ll \tau_{N}
$$

At long times $t \gg \tau_{N}$ the dominating contribution comes from the Maxwellian (retarded) viscosity, and formula (6.2) gives

$$
\left\langle(\Delta \theta)^{2}\right\rangle \simeq \frac{2 T}{\zeta_{M}}\left(t+\tau_{M}\right), \quad t \gg \tau_{N}
$$

where from it follows that in this time interval the orientational diffusion evolves $\tau_{N}<t<\tau_{M}=q \tau_{N}$ times slower than at the short times.

Another important viscoelastic effect is that the transition from the small-scale (fast) to large-scale (slow) diffusion takes place in the time interval $\tau_{N}<t<\tau_{M}=q \tau_{N}$, whose width in real systems may attain several decades. During this process, the phase MSD remains virtually constant:

$$
\frac{1}{2}\left\langle(\Delta \theta)^{2}\right\rangle \simeq \Theta^{2}, \quad \Theta=\frac{q}{1+q} \sqrt{\frac{T}{\kappa_{M}}} .
$$

The cause of this "dynamic localization" (confinement) is that at $t \ll \tau_{M}$ the Maxwell chain reacts to an applied force only with its elastic element. In other words, at the times shorter than $\tau_{M}$, the Jeffreys fluid acts on the particle as if it were an elastic continuum. As seen from Eq. (6.5), that enhanced elasticity reduces the intensity of the particle thermal motion.

It is worth a remark that in polymeric fluids the localization effect should but weakly depend on temperature. This follows from the fact that in those systems, the elasticity modulus is of the entropic origin: $G \sim n T$, where $n$ is the density of physical nodes of the mesh, see [40], for example. The mean distance between these "temporal" links is $l \sim n^{-1 / 3}$. Taking this into account we arrive at the estimate

$$
\left\langle(\Delta \theta)^{2}\right\rangle \simeq(l / a)^{3},
$$

which is temperature independent. Performing similar estimate for the translations motion of a Brownian particle in a viscoelastic fluid, one finds that the spatial scale of the dynamic localization does not depend of temperature and writes

$$
\left\langle(\Delta r)^{2}\right\rangle \simeq l^{3} / a
$$

Therefore, we come to conclusion that the dynamic localization effect is determined, first place, by the mesostructure of the carrier fluid. Besides that, comparison of Eqs. (6.6) and (6.7) implies that the dependence of MSD on the particle size in rotary diffusion is stronger than that in translational Brownian motion. 


\section{Dynamic magnetic susceptibility of a viscoelastic ferrocolloid}

The Brownian motion theory makes the basis for microrheology of complex fluids. This method of mechanical probing of structured fluidic media (polymer solutions, liquid crystals, biological fluids) has two main modifications. One is "passive" microrheology. The measurement is performed by monitoring the motion of a particle(s) inserted in the material under investigation. Here the probing particle is solely driven by thermal motion. In "active" microrheology the particle is set under conditions of forced diffusion so that, along with thermofluctuation forces, it is subjected to some regular stimulus. The most known scheme of active microrheology is registration of rotary (orientational) motion of a dipolar particle in a weak ac field. The vector of the particle dipolar (e.g. magnetic) moment plays the role of orientational marker, and this motion is registered with the aid of induction sensor. Therefore, the main characteristic of interest is the dynamic magnetic susceptibility of the tested sample. It is used for extracting the rheological parameters of the viscoelastic environment of the particle. This enables one to investigate, in particular, opaque samples that is impossible for passive microrheology, whose customary registration techniques is optics. Consider a viscoelastic fluid where to magnetic particles are embedded in an amount, which:

1) does not affect the rheology of the tested medium;

2) allows one to neglect the dipole-dipole and hydrodynamic interactions between the particles;

3) ensures the output signal level minimally sufficient to register the magnetodynamic response.

Upon satisfying these conditions, a viscoelastic magnetic suspension/colloid subjected to a weak linearly polarized magnetic field $H \cos \omega t$, may be treated as a statistical ensemble of non-interacting dipolar particles. In this context, the above-introduced orientational coordinate $\theta$ denotes the angle between the dipolar moment (frozen in the particle body) and the direction of the field polarization.

In the absence of the field, the magnetic moments of the ensemble are oriented at random due to the rotary diffusion, and the observed magnetization of the system is zero. Switching on the field with frequency $\omega$, adds to the orientation motion of the particles a regular component, and induces magnetization (the magnetic moment of unit volume) that oscillates with the frequency of the probing field. To such a situation, the linear response theory applies, which provides a general expression for the susceptibility, see [24], for example. For the ensemble of planar rotators with "frozen" magnetic moments, this expression takes the form

$$
\left.\chi(\omega) \equiv\left(\frac{M_{\omega}}{H_{\omega}}\right)\right|_{H_{\omega} \rightarrow 0}=\chi_{0}\left[1+i \omega \int_{0}^{\infty} d t e^{i \omega t} J(t)\right], \quad J(t)=\frac{\left\langle\cos \theta \cos \theta_{t}\right\rangle}{\left\langle\cos ^{2} \theta\right\rangle}
$$

Here $\chi_{0}=\chi(0)=n \mu^{2} / 2 T$ is the static (equilibrium) susceptibility for this ensemble, $n$ the particle concentration, $J$ is termed dipolar correlation function, and angular brackets denote averaging over the statistical ensemble.

We assume that thermal motion (noise) in the fluid is a "white", that is a Gaussian random process. Then the same applies to the time-dependence of the particle rotation angle $\theta(t)$. Under these conditions, the dipolar correlation function is obtained in explicit form [24,34]:

$$
J(t)=\exp \left[-\frac{1}{2}\left\langle(\Delta \theta)^{2}\right\rangle\right], \quad \Delta \theta=\theta_{t}-\theta .
$$

Substituting this expression to formula (7.1), one gets for the susceptibility

$$
\frac{\chi(\omega)}{\chi_{0}}=1+i \omega \int_{0}^{\infty} d t \exp \left[i \omega t-\frac{t}{(1+q) \tau_{D}}\right] \exp \left[-\Theta^{2}\left(1-e^{-\widetilde{\gamma} t}\right)\right],
$$

where $\tau_{D}=\zeta_{N} / T$ is the Debye time of thermal relaxation that is determined the Newtonian viscosity; the nondimensional temperature $\Theta$ is defined by Eq. (6.5). The integral in formula (7.3) cannot be expressed 
in standard functions, however, on expanding of the integrand in Taylor series with respect to $\Theta$, it may be presented as a sum, where each term is easily integrable. In result, one arrives at the expansion

$$
\frac{\chi(\omega)}{\chi_{0}}=e^{-\Theta^{2}} \sum_{k=0}^{\infty} \frac{\Theta^{2 k}}{k !}\left[1-\frac{i \omega \tau_{D}(1+q)}{1+q k / \Theta^{2}}\right]^{-1},
$$

which is facile for numerical evaluation in all the variation range of the material parameters entering it. According to this formula, the susceptibility of an ensemble of rotatable dipoles is a superposition of relaxators, each of which contributes with the amplitude (relaxator force) determined by its number and the "softness" $\Theta$ of the medium. For a Newtonian fluid $(q \rightarrow 0)$, this formula yields the customary Debye susceptibility

$$
\frac{\chi(\omega)}{\chi_{0}}=\frac{\psi_{D}(\omega)}{\chi_{0}}=\frac{1}{1-i \omega \tau_{D}} .
$$

The character of susceptibility for a colloid with developed viscoelasticity $(q \gg 1)$ is determined mostly by the value of $\Theta$. The relation of this parameter with the mesostructure of the carrier fluid was discussed in the preceding section, see estimate (6.6). From it, it is seen that the dynamic elasticity might be considered in the framework of a continuum media approach only under condition $a \gg l$, i.e., if the size of the probing particle exceeds the reference spatial scale of the mesostructure. At the first sight, it seems that Eq. (7.4) is in inapplicable at $\Theta>1$. However, the latter condition (it is equivalent to $l>a$ ) means that the mesh but slightly affects the particle, the latter does not "feel" the elastic environment. Thence, the main source of friction is the usual Newtonian viscosity, and the dominating motion mode is fast diffusion. Because of that Eq. (7.4), at least qualitatively, holds at arbitrary values of the nondimensional "temperature" $\Theta$.

As Eq. (7.3) shows, under weak elasticity of the fluid $\Theta \gg 1$, i.e., domination of fast diffusion, the level of phase fluctuations is high. In this case $\left\langle(\Delta \theta)^{2}\right\rangle \simeq 2 T t / \zeta_{N}$, see Eq. (6.3), so that integration in Eq. (7.1) yields the Debye susceptibility (7.5). At strong elasticity $(\Theta \ll 1)$, when the leading role is played by slow diffusion, general expression (7.3) again assumes the Debye form but now with another, much greater, time $\tau_{s}=\tau_{D}(1+q) \gg \tau_{D}$. Therefore, in each of the limiting cases, the system is governed by just one of the two possible relaxation mechanisms. Accordingly, in either case the imaginary part of the susceptibility $\chi^{\prime \prime}(\omega)$ has a single-peak shape. In the intermediate situation $(\Theta \sim 1)$, the contributions of both mechanisms are comparable, and in the absorption line (imaginary part of the susceptibility) would comprise both the high-frequency $\omega \sim 1 / \tau_{N}$ and low-frequency $\omega \sim 1 / \tau_{M}$ components.

In Figs. 9-11 solid lines show frequency dependence of the imaginary part of magnetic susceptibility plotted with the aid of Eq. (7.4) in a range of material parameters. Virtually the same curves are obtained, when the calculation is done in some high-order approximation of the moment method, see Appendix 1, Eq. (10.20). Dashed lines in those figures render the results of the "effective field" treatment: when the infinite chain of moment equations (10.11) is truncated and reduced to the first pair of them. In this description, the effect of viscoelasticity on the particle is rendered by just one torque that is linear in the retarded force.

The figures show that, in accordance with the afore-given qualitative analysis, the single-mode relaxation regimes take place at low (panes a in Figs. 9-11) as well as at high temperatures (panes d there). As seen, in these limiting cases the effective field approximation virtually coincides with the exact one. Besides that, Fig. 9 evidences that under moderate viscoelasticity $(q=10)$ the effective field model works fairy well all throughout the temperature range and at any frequency.

The two-mode relaxation regime becomes well-discernible, however, in the systems with developed viscoelasticity at intermediate temperatures $(0.1 \lesssim \Theta \lesssim 1)$, see panes $\mathrm{b}$ and $\mathrm{c}$ in Figs. 10 and 11 . Inside this interval of $\Theta$, the effective-field solution is substantially different from the exact one. Moreover, this difference grows with the nondimensional viscoelasticity parameter $q$, cf. Figs. 10 and 11.

Fig. 12 shows the frequency dependence of the imaginary part of the dynamic susceptibility for an ensemble of rotators at moderate temperatures $0.1 \lesssim \Theta \lesssim 1$. Inside this interval, both diffusion mechanisms contribute to orientation relaxation of the Brownian particle. Their presence is clearly evidenced 

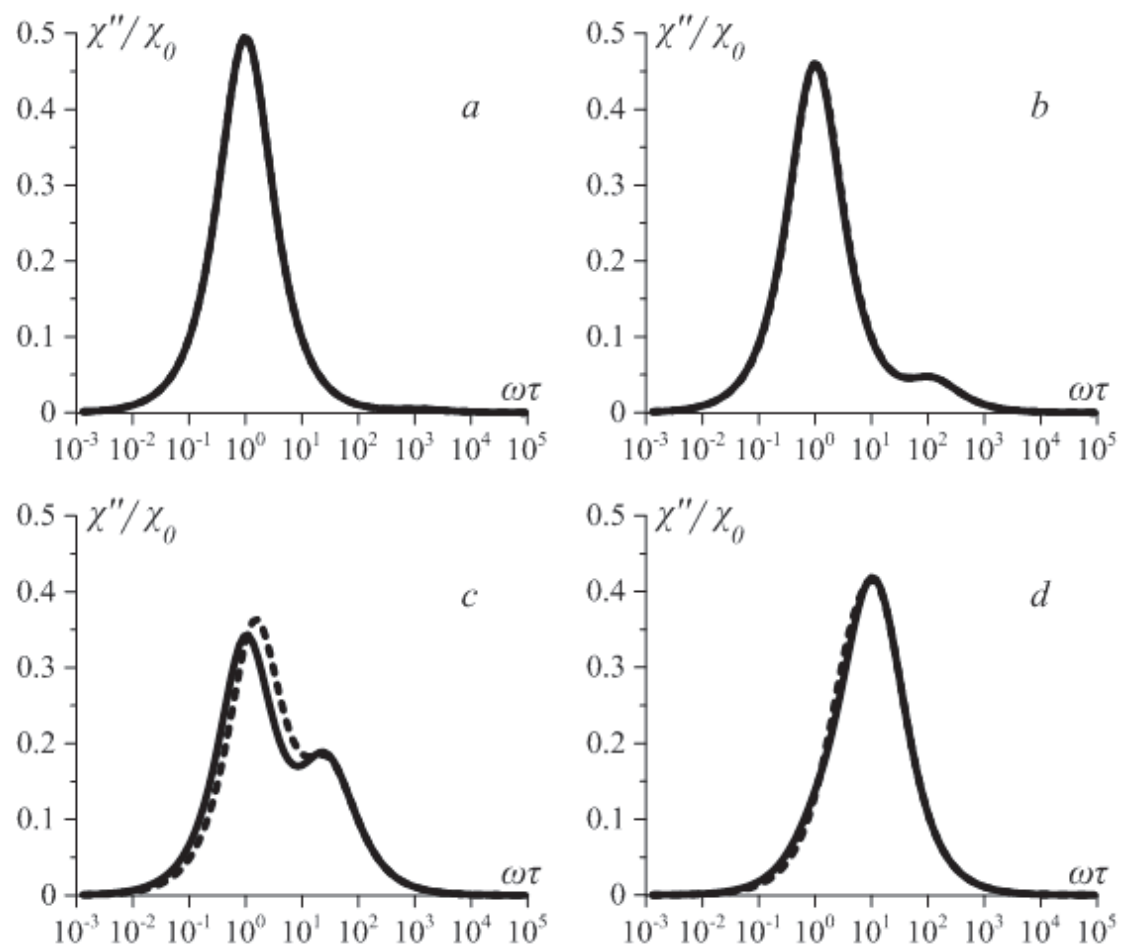

Figure 9. Frequency dependence of imaginary part of the dynamic susceptibility in an ensemble of planar rotators for $q=10$; solid lines are obtained from Eq. (7.4), dashed lines are the result of two-moment (effective-field) approximation of the set (10.11) $\left(M_{2}=0\right)$; the nondimensional temperature is $T / \kappa_{M}=0.01(\mathrm{a}), 0.1(\mathrm{~b}), 0.5(\mathrm{c}), 3(\mathrm{~d})$; the time scale is $\tau=\tau_{D}(1+q)$.

by the two-peak shape of the spectrum. We note also that the high-frequency peak (signature of fast diffusion) grows with temperature, shifting simultaneously to lower frequencies. On the other hand, the low-frequency maximum (signature of slow diffusion) stays put at $\omega \tau=1$ and just diminishes its height.

\section{Dynamic susceptibility of a viscoelastic magnetic colloid. 3D case}

In the preceding section the dynamic susceptibility has been calculated in the framework of a simplified model of orientational motion, viz. in-plane rotation. The solution has been built up with the aid of linear response theory. In the Appendixes it is shown that the same result could be obtained taking as the starting point either the kinetic equation (Appendix I) or the set of Langevin equations (Appendix II). Here we employ the latter method to derive the susceptibility for the case of unrestricted, i.e., 3D, rotations.

The set of equations describing non-inertial orientational motion of a particle in a Jeffreys fluid is

$$
\begin{aligned}
& \dot{e}_{i}=(\boldsymbol{\omega} \times \boldsymbol{e})_{i}, \\
& \omega_{i}=\frac{1}{\zeta_{N}}\left[-\hat{L}_{i} U+Q_{i}+y_{N i}(t)\right], \quad \hat{L}_{i}=\left(\boldsymbol{e} \times \frac{\partial}{\partial \boldsymbol{e}}\right)_{i}, \\
& \dot{Q}_{i}=-\widetilde{\gamma} Q_{i}+\gamma_{N} \hat{L}_{i} U+\kappa_{M}\left[\frac{y_{M}(t)}{\zeta_{M}}-\frac{y_{N}(t)}{\zeta_{N}}\right] .
\end{aligned}
$$

Here $\boldsymbol{e}$ is unit vector of the particle orientation, which we identify with the direction of the dipole, $\boldsymbol{\omega}$ is angular velocity, and $\hat{L}$ operator of infinitesimal rotation. The random forces, which model thermal noise 

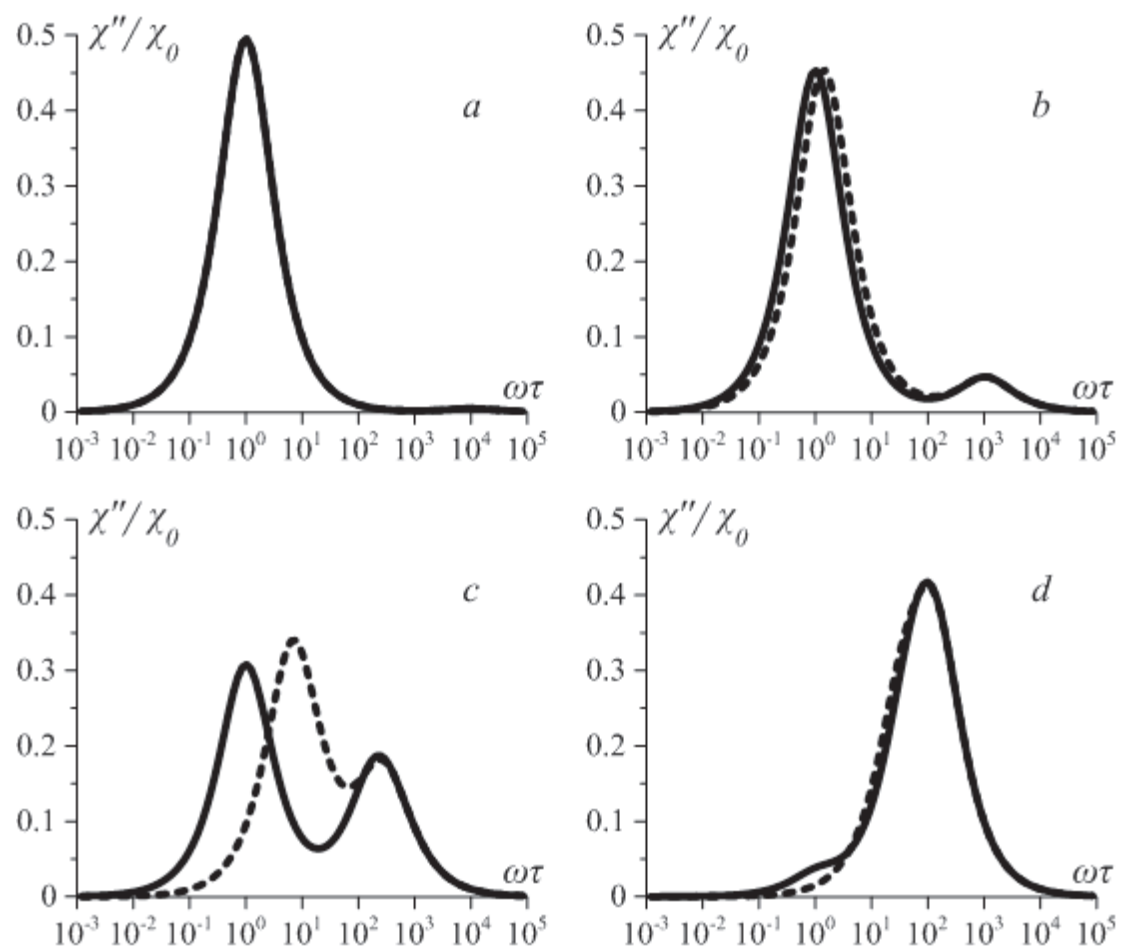

FiguRE 10. Frequency dependence of imaginary part of the dynamic susceptibility in an ensemble of planar rotators for $q=100$; solid lines are obtained from Eq. (7.4), dashed lines are the result of two-moment (effective-field) approximation of the set (10.11) $\left(M_{2}=0\right)$; the nondimensional temperature is $T / \kappa_{M}=0.01(\mathrm{a}), 0.1(\mathrm{~b}), 0.5(\mathrm{c}), 3(\mathrm{~d})$; the time scale is $\tau=\tau_{D}(1+q)$.

in the system, in accordance with the fluctuation-dissipation theorem are defined by the correlator

$$
\left\langle y_{i \alpha}(t) y_{j \beta}(t+\tau)\right\rangle=2 T \zeta_{\alpha} \delta_{\alpha \beta} \delta_{i j} \delta(\tau), \quad \alpha=N, M .
$$

The set of stochastic equations (8.1) might be transformed into a kinetic (Fokker-Planck) equation for the distribution function $W(\boldsymbol{e}, \boldsymbol{Q}, t)$. Performing this as it is has been done before for the case of translational motion (see section 3 ), one gets

$$
\begin{aligned}
\frac{\partial}{\partial t} W+\frac{1}{\zeta_{N}} \hat{L}_{j}\left(Q_{j}+T \kappa_{M} \frac{\partial}{\partial Q_{j}}\right) W & +\gamma_{N} \frac{\partial}{\partial Q_{j}}\left[\left(\hat{L}_{j} U\right)+T \hat{L}_{j}\right] W \\
& =\frac{1}{\zeta_{N}} \hat{L}_{j}\left[\left(\hat{L}_{j} U\right)+T \hat{L}_{j}\right] W+\widetilde{\gamma} \frac{\partial}{\partial Q_{j}}\left(Q_{j}+T \kappa_{M} \frac{\partial}{\partial Q_{j}}\right) W
\end{aligned}
$$

From the structure of this equation it follows that its equilibrium solution is a generalized MaxwellBoltzmann distribution

$$
W_{0}(\boldsymbol{e}, \boldsymbol{Q}) \propto \exp \left\{-\frac{1}{T}\left[U(\boldsymbol{e})+\frac{Q^{2}}{2 \kappa_{M}}\right]\right\} .
$$

In the problem under consideration, the particle dipole moment $\boldsymbol{\mu}$ is fixed in the particle body, so that $\boldsymbol{\mu}=\mu \boldsymbol{e}$. The energy of its interaction with the applied magnetic field has a standard (Zeeman) form

$$
U=-(\boldsymbol{\mu H})=-\mu H(\boldsymbol{e h}), \quad \boldsymbol{h}^{2}=1 .
$$



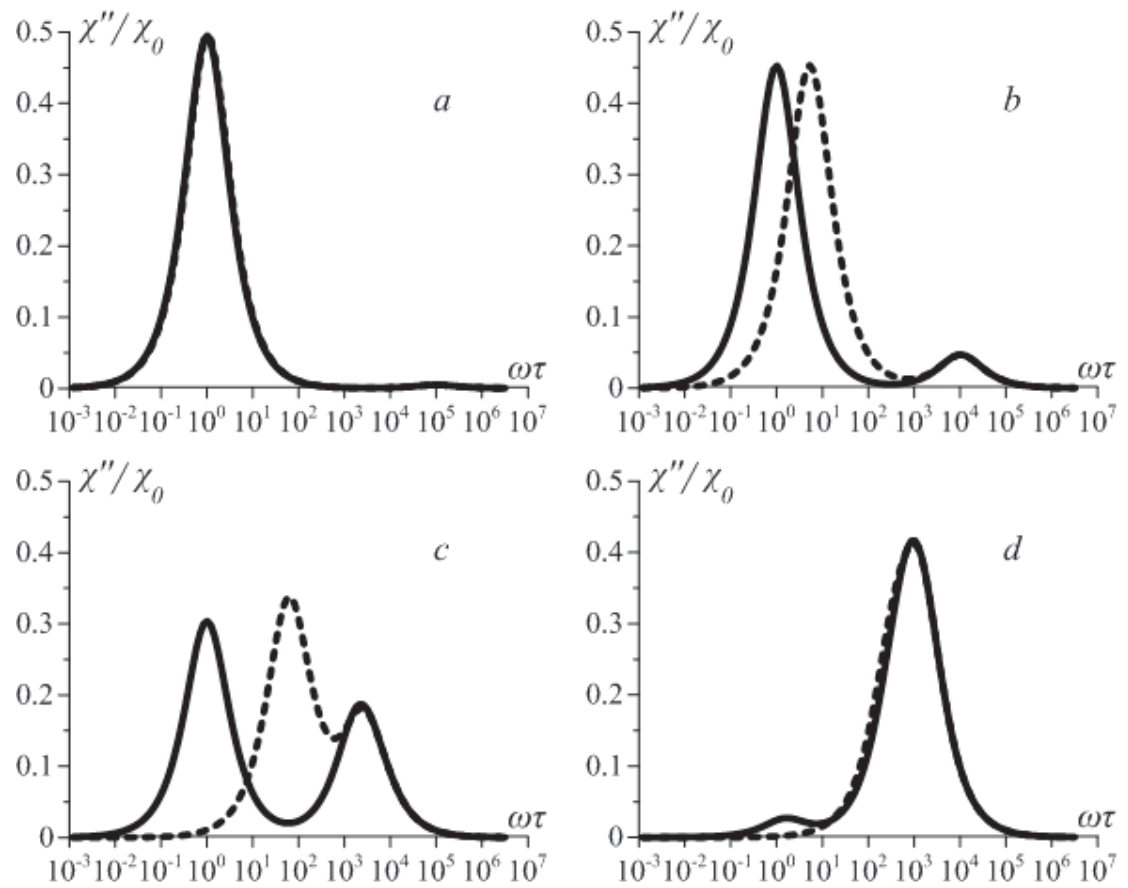

FiguRE 11. Frequency dependence of imaginary part of the dynamic susceptibility in an ensemble of planar rotators for $q=10^{3}$; solid lines are obtained from Eq. (7.4), dashed lines are the result of two-moment (effective-field) approximation of the set (10.11) $\left(M_{2}=0\right)$; the nondimensional temperature is $T / \kappa_{M}=0.01$ (a), $0.1(\mathrm{~b}), 0.5(\mathrm{c}), 3(\mathrm{~d})$; the time scale is $\tau=\tau_{D}(1+q)$.

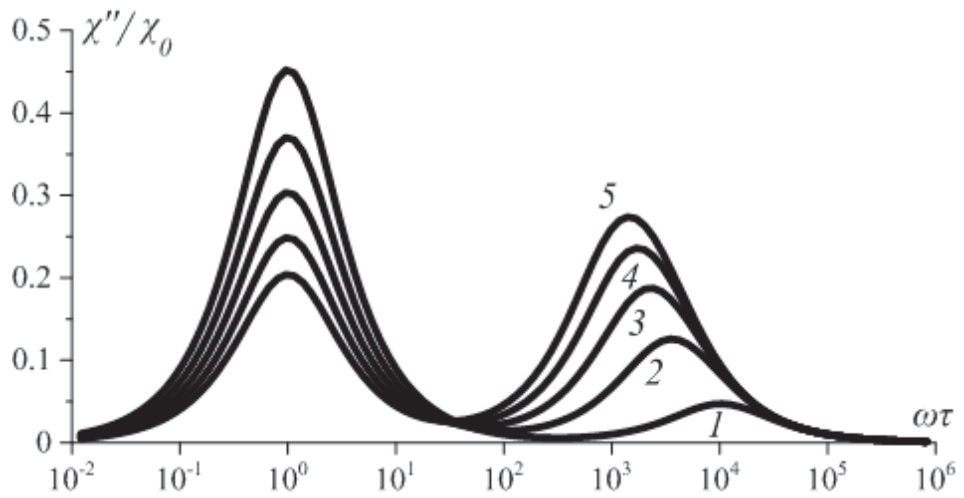

FIGURE 12. Frequency dependence of imaginary part of the dynamic susceptibility in an ensemble of planar rotators for $q=10^{3}$ obtained with Eq. (7.4); the nondimensional temperature is $T / \kappa_{M}=0.1(1), 0.3(2), 0.5(3), 0.7(4), 0.9(5)$.

Let the coordinate axis $O z$ be directed along the field: $\boldsymbol{h}=(0,0,1)$. The sought for quantity $\langle\boldsymbol{e h}\rangle=\left\langle e_{z}\right\rangle$ that is proportional to the magnetization of the system, we evaluate by averaging the Langevin equation (8.1). With allowance for Eq. (8.5), the set writes as

$$
\begin{aligned}
& \dot{e}_{i}=\gamma_{H}(\boldsymbol{e} \times(\boldsymbol{h} \times \boldsymbol{e}))_{i}+\gamma_{N}(\boldsymbol{Q} \times \boldsymbol{e})_{i}+(\boldsymbol{u} \times \boldsymbol{e})_{i}, \\
& \dot{Q}_{i}=-\widetilde{\gamma} Q_{i}-\gamma_{H}(\boldsymbol{e} \times \boldsymbol{h})_{i}+\widetilde{u}_{i} .
\end{aligned}
$$


Here $u_{k} \equiv\left(y_{N}\right)_{k} / \zeta_{N}$ and $\widetilde{u}_{k} \equiv\left(y_{M}\right)_{k} / \zeta_{M}-\left(y_{N}\right)_{k} / \zeta_{N}$, while the torque due to the retarded friction force is put in nondimensional form as $\boldsymbol{Q} \rightarrow \boldsymbol{Q} / \kappa_{M}$. For correlators of the random forces entering this equations one finds using Eq. (8.2):

$$
\begin{aligned}
\left\langle u_{i} u_{j}(\tau)\right\rangle & =\frac{2 T}{\zeta_{N}} \delta_{i j} \delta(\tau), \\
\left\langle u_{i} \widetilde{u}_{j}(\tau)\right\rangle & =\left\langle\widetilde{u}_{i} u_{j}(\tau)\right\rangle=-\frac{2 T}{\zeta_{N}} \delta_{i j} \delta(\tau), \\
\left\langle\widetilde{u}_{i} \widetilde{u}_{j}(\tau)\right\rangle & =\frac{2 T}{\zeta_{N}}\left(1+\frac{1}{q}\right) \delta_{i j} \delta(\tau) .
\end{aligned}
$$

Averaging of the first of equations (8.6) yields

$$
\langle\dot{\boldsymbol{e}}\rangle=\gamma_{H}\langle\boldsymbol{e} \times(\boldsymbol{h} \times \boldsymbol{e})\rangle+\gamma_{N}\langle\boldsymbol{Q} \times \boldsymbol{e}\rangle+\langle\boldsymbol{u} \times \boldsymbol{e}\rangle .
$$

The field term should be averaged with the equilibrium distribution (8.4) setting $U=0$, that renders $\langle\boldsymbol{e} \times(\boldsymbol{h} \times \boldsymbol{e})\rangle_{0}=\frac{2}{3} \boldsymbol{h}$. Using the already described procedure, for the diffusion component of the equation one finds

$$
\langle\boldsymbol{u} \times \boldsymbol{e}\rangle=\hat{I}\left\langle\boldsymbol{u} \times\left(\boldsymbol{u}^{\prime} \times \boldsymbol{e}^{\prime}\right)\right\rangle=\hat{I}\left\langle\boldsymbol{u}^{\prime}\left(\boldsymbol{u} \boldsymbol{e}^{\prime}\right)-\boldsymbol{e}^{\prime}\left(\boldsymbol{u} \boldsymbol{u}^{\prime}\right)\right\rangle=-\frac{2 T}{\zeta_{N}}\langle\boldsymbol{e}\rangle .
$$

In result, the equation for the averaged orientation vector takes the form

$$
\left(\partial / \partial t+\gamma_{D}\right)\langle\boldsymbol{e}\rangle=\frac{2}{3} \gamma_{H} \boldsymbol{h}+\gamma_{N}\langle\boldsymbol{Q} \times \boldsymbol{e}\rangle, \quad \gamma_{D}=2 T / \zeta_{N} .
$$

As seen, the rate of thermal relaxation $\gamma_{D}=2 T / \zeta_{N}$ is twice as large as in the case of plane rotations.

Equation (8.10) contains the torque generated by retarded friction. Let us derive the equation for this variable. Using the easily verified relation $\partial_{t}\langle\boldsymbol{Q} \times \boldsymbol{e}\rangle=\langle\dot{\boldsymbol{Q}} \times \boldsymbol{e}\rangle+\langle\boldsymbol{Q} \times \dot{\boldsymbol{e}}\rangle$, we get

$$
\begin{aligned}
\left(\partial_{t}+\widetilde{\gamma}\right)\langle\boldsymbol{Q} \times \boldsymbol{e}\rangle=\gamma_{H}[\langle\boldsymbol{Q} \times(\boldsymbol{e} \times(\boldsymbol{h} \times \boldsymbol{e}))\rangle & -\langle\boldsymbol{e} \times(\boldsymbol{h} \times \boldsymbol{e})\rangle] \\
& -\gamma_{N}\langle\boldsymbol{Q} \times(\boldsymbol{Q} \times \boldsymbol{e})\rangle+\langle\widetilde{\boldsymbol{u}} \times \boldsymbol{e}\rangle+\langle\boldsymbol{Q} \times(\boldsymbol{u} \times \boldsymbol{e})\rangle .
\end{aligned}
$$

Averaging of the field term over the equilibrium distribution yields $-\frac{2}{3} \gamma_{H} \boldsymbol{h}$, while for the diffusion component one finds

$$
\begin{aligned}
\langle\widetilde{\boldsymbol{u}} \times \boldsymbol{e}\rangle+\langle\boldsymbol{Q} \times & (\boldsymbol{u} \times \boldsymbol{e})\rangle=\hat{I}\left\langle\widetilde{\boldsymbol{u}} \times\left(\boldsymbol{u}^{\prime} \times \boldsymbol{e}^{\prime}\right)+\widetilde{\boldsymbol{u}}^{\prime} \times(\boldsymbol{u} \times \boldsymbol{e})+\boldsymbol{Q} \times\left(\boldsymbol{u} \times\left(\boldsymbol{u}^{\prime} \times \boldsymbol{e}^{\prime}\right)\right)\right\rangle \\
& =\hat{I}\left\langle 2\left[\boldsymbol{u}^{\prime}(\boldsymbol{e} \widetilde{\boldsymbol{u}})-\boldsymbol{e}^{\prime}\left(\widetilde{\boldsymbol{u}} \boldsymbol{u}^{\prime}\right)\right]+\boldsymbol{Q} \times\left[\boldsymbol{u}^{\prime}(\boldsymbol{e} \boldsymbol{u})-\boldsymbol{e}^{\prime}\left(\boldsymbol{u} \boldsymbol{u}^{\prime}\right)\right]\right\rangle=2 \gamma_{D}\langle\boldsymbol{e}\rangle-\gamma_{D}\langle\boldsymbol{Q} \times \boldsymbol{e}\rangle .
\end{aligned}
$$

The resulting equation is

$$
\left(\partial_{t}+\widetilde{\gamma}+\gamma_{D}\right)\langle\boldsymbol{Q} \times \boldsymbol{e}\rangle=-\frac{2}{3} \gamma_{H} \boldsymbol{h}+\gamma_{N}\langle\boldsymbol{Q} \times(\boldsymbol{Q} \times \boldsymbol{e})\rangle+2 \gamma_{D}\langle\boldsymbol{e}\rangle .
$$

Thus we have obtained an equation for the first (with respect to $\boldsymbol{Q}$ ) moment, the right-hand side of which contains the second-order moment. The equation for this latter variable would incorporate the third-order moment and such chaining would occur for all the moments of higher order. In the representation used, the structure of the emerging chain set is rather complicated. It may be simplified considerably by an appropriate choice of the basis functions. This problem is clarified in Appendix II, here we undertake the similar transformations.

From equation (8.6) it follows that it is the torque due to the retarded that "links" the emerging equation chain. Let us show how this works with the increase of the moment order with respect to $\boldsymbol{Q}$. We introduce a set of vector functions

$$
\boldsymbol{N}_{1} \equiv \boldsymbol{Q} \times \boldsymbol{e}, \quad \boldsymbol{N}_{2} \equiv \boldsymbol{Q} \times(\boldsymbol{Q} \times \boldsymbol{e})=\boldsymbol{Q} \times \boldsymbol{N}_{1}, \quad \ldots \quad \boldsymbol{N}_{k+1} \equiv \boldsymbol{Q} \times \boldsymbol{N}_{k},
$$


and expanding the vector product get vector

$$
\boldsymbol{N}_{2}=\boldsymbol{Q}(\boldsymbol{e Q})-e Q^{2},
$$

orthogonal to $\boldsymbol{N}_{1}$, it is easy to see that $\left(\boldsymbol{N}_{1} \boldsymbol{N}_{2}\right)=0$. Vectors $\boldsymbol{N}_{1}$ and $\boldsymbol{N}_{2}$ make a natural basis for the proposed set of vector functions $\left\{\boldsymbol{N}_{k}\right\}$. Indeed, the terms of greater expand easily with respect to this pair:

$$
\begin{aligned}
& \boldsymbol{N}_{3}=\boldsymbol{Q} \times \boldsymbol{N}_{2}=\boldsymbol{Q} \times\left[\boldsymbol{Q}(\boldsymbol{e} \boldsymbol{Q})-\boldsymbol{e} Q^{2}\right]=-Q^{2} \boldsymbol{N}_{1}, \\
& \boldsymbol{N}_{4}=\boldsymbol{Q} \times \boldsymbol{N}_{3}=-Q^{2}\left(\boldsymbol{Q} \times \boldsymbol{N}_{1}\right)=-Q^{2} \boldsymbol{N}_{2}, \\
& \boldsymbol{N}_{2 k+1}=(-1)^{k} Q^{2 k} \boldsymbol{N}_{1}, \quad \boldsymbol{N}_{2 k}=(-1)^{k-1} Q^{2(k-1)} \boldsymbol{N}_{2} .
\end{aligned}
$$

As seen, all the odd in $\boldsymbol{Q}$ moments are collinear with $\boldsymbol{N}_{1}$, and all the even ones are collinear to $\boldsymbol{N}_{2}$. However, vector $\boldsymbol{N}_{2}$ is a sum of two vectors, one of which is collinear with $\boldsymbol{Q}$ and the other to $\boldsymbol{e}$.

For the right choice of the basis, we expand the non-equilibrium distribution function as

$$
\begin{aligned}
W(\boldsymbol{e}, \boldsymbol{Q}, t)=W_{0}(\boldsymbol{Q})[1+\boldsymbol{a}(t) \boldsymbol{e} & +\boldsymbol{b}_{1}(t) \boldsymbol{M}_{1} \\
& \left.+\boldsymbol{b}_{2}(t) \boldsymbol{K}_{2}+\boldsymbol{c}_{2}(t) \boldsymbol{L}_{2}+\boldsymbol{b}_{3}(t) \boldsymbol{M}_{3}+\boldsymbol{b}_{4}(t) \boldsymbol{K}_{4}+\boldsymbol{c}_{4} \boldsymbol{L}_{4}+\ldots\right] .
\end{aligned}
$$

From normalization condition, it follows that

$$
\langle\boldsymbol{e}\rangle_{0}=0, \quad\left\langle\boldsymbol{M}_{1}\right\rangle_{0}=0, \quad\left\langle\boldsymbol{K}_{2}\right\rangle_{0}=0, \quad\left\langle\boldsymbol{L}_{2}\right\rangle_{0}=0, \ldots
$$

Upon imposing on these moments the orthogonality requirement, one gets simple relations for determining the expansion coefficients in Eq. (8.17):

$$
\begin{aligned}
& \langle\boldsymbol{e}\rangle=a(t), \quad\left\langle\boldsymbol{e} \boldsymbol{M}_{1}\right\rangle_{0}=0, \quad\left\langle\boldsymbol{e} \boldsymbol{K}_{2}\right\rangle_{0}=0, \quad\left\langle\boldsymbol{e} \boldsymbol{L}_{2}\right\rangle_{0}=0, \ldots \\
& \boldsymbol{b}_{1}(t)=\frac{\left\langle\boldsymbol{M}_{1}\right\rangle}{\left\langle M_{1}^{2}\right\rangle_{0}},\left\langle\boldsymbol{M}_{1} \boldsymbol{K}_{2}\right\rangle_{0}=0,\left\langle\boldsymbol{M}_{1} \boldsymbol{L}_{2}\right\rangle_{0}=0, \ldots \\
& \boldsymbol{b}_{2}(t)=\frac{\left\langle\boldsymbol{K}_{2}\right\rangle}{\left\langle K_{2}^{2}\right\rangle_{0}},\left\langle\boldsymbol{K}_{2} \boldsymbol{L}_{2}\right\rangle_{0}=0,\left\langle\boldsymbol{K}_{2} \boldsymbol{M}_{3}\right\rangle_{0}=0, \ldots
\end{aligned}
$$

We remind that in these formulas index 0 at the brackets denotes averaging with equilibrium distribution (8.4) (at $U=0$ ), that is

$$
\begin{aligned}
\langle\ldots\rangle_{0}=\frac{1}{(2 \pi \widetilde{T})^{3 / 2}} \int \ldots d^{3} Q e^{-Q^{2} / 2 \widetilde{T}} & \\
= & \frac{1}{(2 \pi \widetilde{T})^{3 / 2}} \int_{-\infty}^{\infty} d Q_{x} \int_{-\infty}^{\infty} d Q_{y} \int_{-\infty}^{\infty} d Q_{z} \ldots e^{-\left(Q_{x}^{2}+Q_{y}^{2}+Q_{z}^{2}\right) / 2 \widetilde{T}}, \quad \widetilde{T}=T / \kappa_{M} .
\end{aligned}
$$

The structure of vector $\boldsymbol{N}_{2}$ implies that one of the even second-order basis functions may be present in the form $\widetilde{\boldsymbol{K}}_{2}=\boldsymbol{e} Q^{2}$. To transform function $\widetilde{\boldsymbol{K}}_{2}$ into one that is orthogonal to the first basis function of expansion (8.17), we expand function $\boldsymbol{K}_{2}$ as

$$
\boldsymbol{K}_{2}=\widetilde{\boldsymbol{K}}_{2}+A \boldsymbol{e}=\boldsymbol{e}\left(Q^{2}+A\right) .
$$

From the condition of statistical orthogonality $\left\langle\boldsymbol{K}_{2} \boldsymbol{e}\right\rangle_{0}=0$, we get

$$
\boldsymbol{K} \equiv \boldsymbol{K}_{2}=e\left(Q^{2}-3 \widetilde{T}\right)
$$

Another second-order vector function we present as

$$
\boldsymbol{L} \equiv \boldsymbol{L}_{2}=\boldsymbol{Q}(\boldsymbol{e Q})-\frac{1}{3} \boldsymbol{e} Q^{2}
$$


which ensures its orthogonality to vector $\boldsymbol{e}$ and, accordingly, to function $\boldsymbol{K}_{2}$. Give that, vector $\boldsymbol{N}_{2}$ could be presented in the form

$$
\boldsymbol{N}_{2}=\boldsymbol{Q}(\boldsymbol{e Q})-\boldsymbol{e} Q^{2} \equiv \boldsymbol{L}_{2}-\frac{2}{3} \widetilde{\boldsymbol{K}}_{2}, \quad \widetilde{\boldsymbol{K}}_{2}=e Q^{2} .
$$

The above-derived basis functions split in three series, viz. $\left\{\boldsymbol{M}_{k}\right\},\left\{\boldsymbol{L}_{k}\right\}$ and $\left\{\boldsymbol{K}_{k}\right\}$. The first of them, based on vector $\boldsymbol{N}_{1}=\boldsymbol{Q} \times \boldsymbol{e}$, is orthogonal to the two others, $\left\{\boldsymbol{L}_{k}\right\}$ and $\left\{\boldsymbol{K}_{k}\right\}$, built on vectors $\boldsymbol{e}$ and $\boldsymbol{Q}$, respectively, due to the fundamental property of vector product: $\boldsymbol{e} \cdot(\boldsymbol{Q} \times \boldsymbol{e})=\boldsymbol{Q} \cdot(\boldsymbol{Q} \times \boldsymbol{e}) \equiv 0$. The sets $\left\{\boldsymbol{L}_{k}\right\}$ and $\left\{\boldsymbol{K}_{k}\right\}$ are mutually orthogonal because the ground state of the system is isotropic: $\left\langle e_{i} e_{j}\right\rangle_{0}=\frac{1}{3} \delta_{i j}$. Inside each of the series, a set of orthogonal functions of argument $\boldsymbol{Q}$ is constructed.

We demonstrate the orthogonalization procedure taking first basis functions as examples. Consider the basis function, which is of the third order in $\boldsymbol{Q}$ :

$$
\boldsymbol{M}_{3}=\boldsymbol{N}_{3}+A \boldsymbol{N}_{1}=\left(-Q^{2}+A\right) \boldsymbol{N}_{1} .
$$

The orthogonality of $\boldsymbol{M}_{3}$ to the first (in $\boldsymbol{Q}$ ) basis function yields the expression for coefficient $A$ :

$$
A=\frac{\left\langle Q^{2} N_{1}^{2}\right\rangle_{0}}{\left\langle N_{1}^{2}\right\rangle_{0}} .
$$

Since we denote all the odd-order (with respect to $\boldsymbol{Q}$ ) functions as $\boldsymbol{M}$, in what follows, to shorten notations, we set $M \equiv N_{1}=Q \times e$. The averaged value of the squared basis function $M$ is

$$
\begin{aligned}
\left\langle M^{2}\right\rangle_{0}=\left\langle(\boldsymbol{e} \times \boldsymbol{Q})^{2}\right\rangle & =\varepsilon_{i j k} \varepsilon_{i m n}\left\langle e_{j} e_{m} Q_{k} Q_{n}\right\rangle_{0} \\
& =\frac{1}{3}\left(\delta_{j m} \delta_{k n}-\delta_{j n} \delta_{k m}\right) \delta_{j m}\left\langle Q_{k} Q_{n}\right\rangle_{0}=\frac{2}{3}\left\langle Q^{2}\right\rangle_{0} \equiv 2 \widetilde{T} ;
\end{aligned}
$$

here vector product is written in terms of Levi-Civitta symbols. When averaging over the equilibrium state, see Eq. (8.20), due to isotropy, all the odd-order moments vanish, while the even ones are rendered by simple formula $\left\langle Q^{2 n}\right\rangle_{0}=\widetilde{T}^{n}(2 n+1)$ !!. Evaluating the numerator in the expression of coefficient $A$ in Eq. (8.26) as

$$
\left\langle Q^{2} M^{2}\right\rangle_{0}=\frac{2}{3}\left\langle Q^{4}\right\rangle_{0}=10 \widetilde{T}^{2},
$$

one gets the third-order orthogonal basis function:

$$
M_{3}=N_{3}+5 \widetilde{T} M=\left(-Q^{2}+5 \widetilde{T}\right) M
$$

The fifth-order basis function is sought in the form

$$
M_{5}=N_{5}+A M_{3}+B M_{1}=Q^{4} M_{1}+A M_{3}+B M_{1} .
$$

The normalizing coefficients $(A, B)$ are determined from the orthogonality conditions

$$
\left\langle M_{5} M_{1}\right\rangle=0, \quad\left\langle M_{5} M_{3}\right\rangle=0,
$$

where from we obtain

$$
A=-\frac{\left\langle\boldsymbol{N}_{5} \boldsymbol{M}_{3}\right\rangle_{0}}{\left\langle M_{3}^{2}\right\rangle_{0}}, \quad B=-\frac{\left\langle\boldsymbol{N}_{5} \boldsymbol{M}_{1}\right\rangle_{0}}{\left\langle M_{1}^{2}\right\rangle_{0}} .
$$

Non-orthogonal even-order functions are defined by relations

$$
\widetilde{\boldsymbol{K}}_{2 k}=\boldsymbol{e} Q^{2 k}, \quad \widetilde{\boldsymbol{L}}_{2 k}=\boldsymbol{L} \cdot Q^{2(k-1)} .
$$

We remind that these sets are orthogonal to each other as well as to the set $\left\{\boldsymbol{M}_{2 k+1}\right\}$. Therefore, orthogonalization should be performed only inside each of the set. This is done in a standard way. For example,

$$
\boldsymbol{K}_{4}=\widetilde{\boldsymbol{K}}_{4}+A \boldsymbol{K}_{2}+B \boldsymbol{e}
$$


For coefficients $A$ and $B$ we require $\left\langle\boldsymbol{K}_{4} \boldsymbol{e}\right\rangle_{0}=\left\langle\boldsymbol{K}_{4} \boldsymbol{K}_{2}\right\rangle_{0}=0$ that yields

$$
A=-\frac{\left\langle\widetilde{\boldsymbol{K}}_{4} \boldsymbol{K}_{2}\right\rangle_{0}}{\left\langle K_{2}^{2}\right\rangle_{0}}, \quad B=-\left\langle\widetilde{\boldsymbol{K}}_{4} \boldsymbol{e}\right\rangle_{0} .
$$

The second set of even function is treated in the same way. To show the result explicitly, below we present several first basis functions transformed by the above-described procedure:

$$
\begin{aligned}
& \boldsymbol{M}=\boldsymbol{N}_{1}=\boldsymbol{Q} \times \boldsymbol{e}, \quad \boldsymbol{N}_{k+1} \equiv \boldsymbol{Q} \times \boldsymbol{N}_{k}, \quad \boldsymbol{N}_{0}=\boldsymbol{e}, \\
& \left\langle M^{2}\right\rangle_{0}=2 \widetilde{T}, \\
& \boldsymbol{K} \equiv \boldsymbol{K}_{2}=\widetilde{\boldsymbol{K}}_{2}-3 \widetilde{T} \boldsymbol{e}=\left(Q^{2}-3 \widetilde{T}\right) \boldsymbol{e}, \\
& \left\langle K^{2}\right\rangle_{0}=6 \widetilde{T} \text {, } \\
& \boldsymbol{L} \equiv \boldsymbol{L}_{2}=\boldsymbol{Q}(\boldsymbol{e} \boldsymbol{Q})-\frac{1}{3} \boldsymbol{e} Q^{2}, \\
& \boldsymbol{M}_{3}=\boldsymbol{N}_{3}+5 \widetilde{T} \boldsymbol{M}=\left(-Q^{2}+5 \widetilde{T}\right) \boldsymbol{M}, \\
& \left\langle L^{2}\right\rangle_{0}=\frac{10}{3} \widetilde{T}^{2}, \\
& \boldsymbol{K}_{4}=\widetilde{\boldsymbol{K}}_{4}-10 \widetilde{T} \boldsymbol{K}_{2}-15 \widetilde{T}^{2} \boldsymbol{e}=\left(Q^{4}-10 \widetilde{T} Q^{2}+15 \widetilde{T}^{2}\right) \boldsymbol{e}, \\
& \left\langle M_{3}^{2}\right\rangle_{0}=20 \widetilde{T}^{3}, \\
& \boldsymbol{L}_{4}=\widetilde{\boldsymbol{L}}_{4}-7 \widetilde{T} \boldsymbol{L}=\left(Q^{2}-7 \widetilde{T}\right) \boldsymbol{L}, \\
& \left\langle K_{4}^{2}\right\rangle_{0}=120 \widetilde{T}^{4}, \\
& \boldsymbol{M}_{5}=\boldsymbol{N}_{5}+14 \widetilde{T} \boldsymbol{M}_{3}-35 \widetilde{T}^{2} \boldsymbol{M}=\left(Q^{4}-14 \widetilde{T} Q^{2}+35 \widetilde{T}^{2}\right) \boldsymbol{M} \\
& \left\langle L_{4}^{2}\right\rangle_{0}=\frac{140}{3} \widetilde{T}^{4}, \\
& \boldsymbol{K}_{6}=\widetilde{\boldsymbol{K}}_{6}-21 \widetilde{T} \boldsymbol{K}_{4}-105 \widetilde{T}^{2} \boldsymbol{K}_{2}-105 \widetilde{T}^{3} \boldsymbol{e}, \\
& \boldsymbol{L}_{6}=\widetilde{\boldsymbol{L}}_{6}-18 \widetilde{T} \boldsymbol{L}_{4}-63 \widetilde{T}^{2} \boldsymbol{L}, \\
& \boldsymbol{M}_{7}=\boldsymbol{N}_{7}+27 \widetilde{T} \boldsymbol{M}_{5}-189 \widetilde{T}^{2} \boldsymbol{M}_{3}+315 \widetilde{T}^{3} \boldsymbol{M} .
\end{aligned}
$$

This gives the functional basis that simplifies the chain-like set of the moment equations. In above, the first two of these equations have been obtained, see (8.11) and (8.13). However, the second of them contains a non-orthogonalized second-order moment. We transform it to orthogonalized form using Eq. (8.36):

$$
\langle\boldsymbol{Q} \times(\boldsymbol{Q} \times \boldsymbol{e})\rangle=\left\langle\boldsymbol{N}_{2}\right\rangle=\left\langle\boldsymbol{L}_{2}-\frac{2}{3} \widetilde{\boldsymbol{K}}_{2}\right\rangle=\left\langle\boldsymbol{L}_{2}-\frac{2}{3} \boldsymbol{K}_{2}-2 \widetilde{T} \boldsymbol{e}\right\rangle .
$$

Substitution of this in Eq. (8.13) with allowance $\gamma_{D}=2 \widetilde{T} \gamma_{N}=2 T / \zeta_{N}$ gives

$$
\left(\partial_{t}+\widetilde{\gamma}+\gamma_{D}\right)\langle\boldsymbol{M}\rangle=-\frac{2}{3} \gamma_{H} \boldsymbol{h}+\gamma_{N}\left\langle\boldsymbol{L}_{2}-\frac{2}{3} \boldsymbol{K}_{2}\right\rangle+\gamma_{D}\langle\boldsymbol{e}\rangle .
$$

Construction of the next equation of the chain we begin from the equation for non-orthogonalized secondorder moment $\boldsymbol{N}_{2}$. Since $\partial_{t}\left\langle\boldsymbol{N}_{2}\right\rangle=\langle\dot{\boldsymbol{Q}} \times(\boldsymbol{Q} \times \boldsymbol{e})+\boldsymbol{Q} \times(\dot{\boldsymbol{Q}} \times \boldsymbol{e})+\boldsymbol{Q} \times(\boldsymbol{Q} \times \dot{\boldsymbol{e}})\rangle$, then, using the Langevin equations (8.6), we find

$$
\begin{aligned}
\left(\partial_{t}+2 \widetilde{\gamma}\right)\left\langle\boldsymbol{N}_{2}\right\rangle=\gamma_{H}\langle\boldsymbol{Q} \times(\boldsymbol{Q} & \times(\boldsymbol{e} \times(\boldsymbol{h} \times \boldsymbol{e}))) \\
& -\boldsymbol{e} \times(\boldsymbol{h} \times(\boldsymbol{Q} \times \boldsymbol{e}))-\boldsymbol{Q} \times(\boldsymbol{e} \times(\boldsymbol{h} \times \boldsymbol{e}))\rangle_{0}+\gamma_{N}\left\langle\boldsymbol{Q} \times \boldsymbol{N}_{2}\right\rangle_{0}+\hat{\boldsymbol{D}} .
\end{aligned}
$$

Here the diffusion component has the form

$$
\hat{D}=\langle\widetilde{\boldsymbol{u}} \times(\boldsymbol{Q} \times \boldsymbol{e})+\boldsymbol{Q} \times(\widetilde{\boldsymbol{u}} \times \boldsymbol{e})+\boldsymbol{Q} \times(\boldsymbol{Q} \times(\boldsymbol{u} \times \boldsymbol{e}))\rangle .
$$

Calculating it, as in above, we arrive at

$$
\begin{aligned}
\left.\hat{\boldsymbol{D}}=\hat{I}\left\langle\widetilde{\boldsymbol{u}} \times \widetilde{\boldsymbol{u}}^{\prime} \times \boldsymbol{e}\right)\right)+\widetilde{\boldsymbol{u}} \times\left(\boldsymbol{Q} \times\left(\boldsymbol{u}^{\prime} \times \boldsymbol{e}^{\prime}\right)\right)+\widetilde{\boldsymbol{u}}^{\prime} \times(\widetilde{\boldsymbol{u}} \times \boldsymbol{e})+\boldsymbol{Q} \times\left(\widetilde{\boldsymbol{u}} \times\left(\boldsymbol{u}^{\prime} \times \boldsymbol{e}^{\prime}\right)\right) \\
+\widetilde{\boldsymbol{u}}^{\prime} \times(\boldsymbol{Q} \times(\boldsymbol{u} \times \boldsymbol{e}))+\boldsymbol{Q} \times\left(\widetilde{\boldsymbol{u}}^{\prime} \times(\boldsymbol{u} \times \boldsymbol{e})\right)+\boldsymbol{Q} \times\left(\boldsymbol{Q} \times\left(\boldsymbol{u} \times\left(\boldsymbol{u}^{\prime} \times \boldsymbol{e}^{\prime}\right)\right)\right\rangle \\
=\hat{I}\left\langle 2\left[\left(\widetilde{\boldsymbol{u}}^{\prime} \widetilde{\boldsymbol{u}}\right)-\boldsymbol{e}\left(\widetilde{\boldsymbol{u}}^{\prime} \widetilde{\boldsymbol{u}}\right)+\widetilde{\boldsymbol{u}} \times \widetilde{\boldsymbol{u}}^{\prime}\left(\boldsymbol{e}^{\prime} \boldsymbol{Q}\right)-\boldsymbol{e}^{\prime}\left(\boldsymbol{u}^{\prime} \boldsymbol{Q}\right)\right]\right. \\
\left.+2 \boldsymbol{Q} \times\left[\boldsymbol{u}^{\prime}\left(\boldsymbol{e}^{\prime} \widetilde{\boldsymbol{u}}\right)-\boldsymbol{e}^{\prime}\left(\widetilde{\boldsymbol{u}} \boldsymbol{u}^{\prime}\right)\right]+\boldsymbol{Q} \times\left(\boldsymbol{Q} \times\left[\boldsymbol{u}^{\prime}\left(\boldsymbol{e}^{\prime} \boldsymbol{u}\right)-\boldsymbol{e}^{\prime}\left(\boldsymbol{u} \boldsymbol{u}^{\prime}\right)\right]\right)\right\rangle .
\end{aligned}
$$


This expression is substituted in the random force correlators (8.7) and then integrated according to definition (4.5). On evaluation of the coefficient at $\gamma_{H}$ and with the result (8.41) for $\hat{\boldsymbol{D}}$, equation (8.40) takes the form

$$
\left(\partial_{t}+2 \widetilde{\gamma}+\gamma_{D}\right)\left\langle\boldsymbol{N}_{2}\right\rangle=-\frac{4}{3} \widetilde{T} \gamma_{H} \boldsymbol{h}+\gamma_{N}\left\langle\boldsymbol{N}_{3}\right\rangle+3 \gamma_{D}\left\langle\boldsymbol{M}_{1}\right\rangle-2 \gamma_{D}\left(1+q^{-1}\right)\langle\boldsymbol{e}\rangle .
$$

that needs orthogonalization. According to definition (8.24), one has the expansion

$$
\boldsymbol{N}_{2} \equiv \boldsymbol{Q}(\boldsymbol{e} \boldsymbol{Q})-\boldsymbol{e} Q^{2}=\boldsymbol{L}_{2}-\frac{2}{3} \boldsymbol{K}_{2}-2 \widetilde{T} \boldsymbol{e} .
$$

Substituting this in the equation for $\left\langle\boldsymbol{N}_{2}\right\rangle$ and expressing, in its right-hand part, non-orthogonalized function $\boldsymbol{N}_{3}$ is expressed (see (8.29)) through orthogonalized $\boldsymbol{M}_{3}$ and $\boldsymbol{M}$, we get

$$
\left(\partial_{t}+2 \widetilde{\gamma}+\gamma_{D}\right)\left\langle\boldsymbol{L}_{2}-\frac{2}{3} \boldsymbol{K}_{2}\right\rangle=\frac{3}{2} \gamma_{D}\left\langle\boldsymbol{M}_{1}\right\rangle+\gamma_{N}\left\langle\boldsymbol{M}_{3}\right\rangle .
$$

To derive the equation for each of two even moments, one more equation is needed. It is convenient to obtain equation for $\left\langle\widetilde{\boldsymbol{K}}_{2}\right\rangle$. After standard transformation to orthogonalized moments, we arrive at

$$
\left(\partial_{t}+2 \widetilde{\gamma}+\gamma_{D}\right)\left\langle\boldsymbol{K}_{2}\right\rangle=-\gamma_{D}\left\langle\boldsymbol{M}_{1}\right\rangle-\gamma_{N}\left\langle\boldsymbol{M}_{3}\right\rangle
$$

At the next step of constructing the chain, the equation for $\left\langle\boldsymbol{M}_{3}\right\rangle$ is derived and then those for $\left\langle\boldsymbol{K}_{4}\right\rangle$ and $\left\langle\boldsymbol{L}_{4}\right\rangle$. In this manner, the chain could be enlarged sequentially to a given order. We have done that up to sixth order, the result is expressed in terms of projections of the corresponding equations on the direction of the probing field $\boldsymbol{h}=(0,0,1)$; to make it more compact, the projection index is omitted:

$$
\begin{aligned}
& \left(\partial_{t}+\Gamma_{0}\right)\left\langle e_{z}\right\rangle=\frac{2}{3} \gamma_{H}+\gamma_{N}\left\langle M_{1}\right\rangle, \\
& \left(\partial_{t}+\Gamma_{1}\right)\left\langle M_{1}\right\rangle=-\frac{2}{3} \gamma_{H}+\gamma_{N}\left\langle L_{2}-\frac{2}{3} K_{2}\right\rangle+\gamma_{D}\left\langle e_{z}\right\rangle, \\
& \left(\partial_{t}+\Gamma_{2}\right)\left\langle K_{2}\right\rangle=-\gamma_{D}\left\langle M_{1}\right\rangle-\gamma_{N}\left\langle M_{3}\right\rangle, \\
& \left(\partial_{t}+\Gamma_{2}\right)\left\langle L_{2}\right\rangle=\frac{5}{6} \gamma_{D}\left\langle M_{1}\right\rangle+\frac{1}{3} \gamma_{N}\left\langle M_{3}\right\rangle, \\
& \left(\partial_{t}+\Gamma_{3}\right)\left\langle M_{3}\right\rangle=\gamma_{D}\left\langle L_{2}-\frac{5}{3} K_{2}\right\rangle+\gamma_{N}\left\langle-L_{4}+\frac{2}{3} K_{4}\right\rangle, \\
& \left(\partial_{t}+\Gamma_{4}\right)\left\langle K_{4}\right\rangle=2 \gamma_{D}\left\langle M_{3}\right\rangle+\gamma_{N}\left\langle M_{5}\right\rangle, \\
& \left(\partial_{t}+\Gamma_{4}\right)\left\langle L_{4}\right\rangle=-\frac{7}{6} \gamma_{D}\left\langle M_{3}\right\rangle-\frac{1}{3} \gamma_{N}\left\langle M_{5}\right\rangle \\
& \left(\partial_{t}+\Gamma_{5}\right)\left\langle M_{5}\right\rangle=\gamma_{D}\left\langle-2 L_{4}+\frac{7}{3} K_{4}\right\rangle+\gamma_{N}\left\langle L_{6}-\frac{2}{3} K_{6}\right\rangle, \\
& \left(\partial_{t}+\Gamma_{6}\right)\left\langle K_{6}\right\rangle=-3 \gamma_{D}\left\langle M_{5}\right\rangle-\gamma_{N}\left\langle M_{7}\right\rangle, \\
& \left(\partial_{t}+\Gamma_{6}\right)\left\langle L_{6}\right\rangle=\frac{3}{2} \gamma_{D}\left\langle M_{5}\right\rangle+\frac{1}{3} \gamma_{N}\left\langle M_{7}\right\rangle, \\
& \Gamma_{n} \equiv \gamma_{D}+n \tilde{\gamma}=\gamma_{D}+n\left(\gamma_{M}+\gamma_{N}\right) .
\end{aligned}
$$

To reveal the structure of the set, we write it in matrix form and specialize it to describe the response to a harmonic signal of frequency $\omega$. For simplicity of notations, we denote the corresponding components of the response by the same symbols omit the index $\omega$. The equations are made nondimensional, for which purpose they are multiplied by the Debye time $\tau_{D}=\zeta_{N} / 2 T$. Then the set (8.46) becomes a matrix equation:

$$
A_{i j} x_{j}=y_{i}
$$

where the vector of the sought for components and that of the external force are defined as follows (sign "T" means transposition):

$$
\begin{aligned}
& x_{j}^{\mathrm{T}}=\left(e, M, K_{2}, L_{2}, M_{3}, K_{4}, L_{4}, M_{5}, K_{6}, L_{6}, \ldots\right) \\
& y_{j}^{\mathrm{T}}=\frac{1}{3} \xi(1,-1,0,0, \ldots, 0, \ldots)
\end{aligned}
$$

and the parameters are

$$
g_{n} \equiv \Gamma_{n} \tau_{D}-i \omega \tau_{D}=1+\frac{1}{2} n \alpha \beta-i \omega \tau_{D}, \quad \beta=\kappa_{M} / T=1 / \widetilde{T}, \quad \alpha=1+1 / q .
$$


Then matrix $A$ takes the form

$$
\left(\begin{array}{ccccccccccc}
g_{0} & -\frac{1}{2} \beta & 0 & 0 & 0 & 0 & 0 & 0 & 0 & 0 & \\
-1 & g_{1} & \frac{1}{3} \beta & -\frac{1}{2} \beta & 0 & 0 & 0 & 0 & 0 & 0 & \\
0 & 1 & g_{2} & 0 & \frac{1}{2} \beta & 0 & 0 & 0 & 0 & 0 & 0 \\
0 & -\frac{5}{6} & 0 & g_{2} & -\frac{1}{6} \beta & 0 & 0 & 0 & 0 & 0 & 0 \\
0 & 0 & \frac{5}{3} & -1 & g_{3} & -\frac{1}{3} \beta & \frac{1}{2} \beta & 0 & 0 & 0 & 0 \\
0 & 0 & 0 & 0 & -2 & g_{4} & 0 & -\frac{1}{2} \beta & 0 & 0 & 0 \\
0 & 0 & 0 & 0 & \frac{7}{6} & 0 & g_{4} & \frac{1}{6} \beta & 0 & 0 & 0 \\
0 & 0 & 0 & 0 & 0 & -\frac{7}{3} & 2 & g_{5} & \frac{1}{3} \beta & -\frac{1}{2} \beta & 0 \\
0 & 0 & 0 & 0 & 0 & 0 & 0 & 3 & g_{6} & 0 & \frac{1}{2} \beta \\
0 & 0 & 0 & 0 & 0 & 0 & 0 & -\frac{9}{6} & 0 & g_{6} & -\frac{1}{6} \beta \\
0 & 0 & 0 & 0 & 0 & 0 & 0 & 0 & \frac{9}{3} & -3 & g_{7}
\end{array}\right)
$$

As seen, it has a simple, almost band-like, structure. Moreover, from this view it is easy to find the algorithm of its continuation to arbitrary rank.

The obtained set could be presented as a five-term recurrence relation

$$
C_{n}^{-} x_{n-2}+B_{n}^{-} x_{n-1}+A_{n} x_{n}+B_{n}^{+} x_{n+1}+C_{n}^{+} x_{n+2}=f_{n} .
$$

It solution is found by the backward-sweep method, see [41], for example. Introducing sweeping coefficients and the free term as

$$
x_{n}=S_{n} x_{n-1}+P_{n} x_{n-2}+a_{n},
$$

one gets

$$
\begin{aligned}
x_{n+1}= & S_{n+1} x_{n}+P_{n+1} x_{n-1}+a_{n+1}, \\
x_{n+2}= & S_{n+2} x_{n+1}+P_{n+2} x_{n}+a_{n+2} \\
& =\left(S_{n+2} S_{n+1}+P_{n+2}\right) x_{n}+S_{n+2} P_{n+1} x_{n-1}+a_{n+2}+S_{n+2} a_{n+1} .
\end{aligned}
$$

Substituting these expressions in the initial equation, one arives at a three-term relation. Comparing it to definition (8.52) of the sweeping parameters $(S, P)$, we find

$$
\begin{aligned}
& P_{n}=-C_{n}^{-} / Z_{n}, \quad Z_{n}=A_{n}+B_{n}^{+} S_{n+1}+C_{n}^{+}\left(S_{n+2} S_{n+1}+P_{n+2}\right), \\
& S_{n}=-\left(B_{n}^{-}+B_{n}^{+} P_{n+1}+C_{n}^{+} S_{n+2} P_{n+1}\right) / Z_{n}, \\
& a_{n}=\left[f_{n}-B_{n}^{+} a_{n+1}-C_{n}^{+}\left(S_{n+2} a_{n+1}+a_{n+2}\right)\right] / Z_{n} .
\end{aligned}
$$

The afore-given view of matrix $A$ implies that the coefficients of the recurrence equation could be written in vector form

$$
\begin{aligned}
& \boldsymbol{A}=\left(g_{0}, g_{1}, g_{2}, g_{2}, g_{3}, g_{4}, g_{4}, g_{5}, g_{6}, g_{6}, \ldots\right) \\
& \boldsymbol{B}^{-}=(0,-1,1,0,-1,-2,0,2,3,0,-3,-4,0,4,5, \ldots) ; \\
& \boldsymbol{C}^{-}=\left(0,0,0,-\frac{5}{6}, \frac{5}{3}, 0, \frac{7}{6},-\frac{7}{3}, 0,-\frac{9}{6}, \frac{9}{3}, 0, \frac{11}{6},-\frac{11}{3}, \ldots\right) ; \\
& \boldsymbol{C}^{+}=\frac{1}{2} \beta(0,-1,1,0,1,-1,0,-1,1,0,1,-1,0,-1,1,0,1,-1,0, \ldots) ; \\
& \boldsymbol{B}^{+}=\frac{1}{6} \beta(-3,2,0,-1,-2,0,1,2,0,-1,-2,0,1,2,0, \ldots) ; \\
& f=\frac{1}{3} \xi(1,-1,0,0, \ldots, 0, \ldots) .
\end{aligned}
$$

Our goal is the dynamic magnetization of the system. The first equation of the chain is

$$
g_{0} x_{1}-\frac{1}{2} \beta x_{2}=\frac{1}{3} \xi
$$

From the definition of the sweeping coefficients (8.52) one has

$$
x_{2}=S_{2} x_{1}+a_{2}
$$


where the free term is found from Eq. (8.54) and equals $a_{2}=-\xi / 3 Z_{2}$. Using these expressions in Eq. (8.56), we get a simple result

$$
x_{1}=\left\langle e_{z}\right\rangle_{\omega}=\langle(\boldsymbol{e h})\rangle_{\omega}=\frac{1}{3} \xi\left(1-\frac{\beta}{2 Z_{2}}\right)\left(1-i \omega \tau_{D}-\frac{1}{2} \beta S_{2}\right)^{-1} .
$$

Thence for evaluation of the susceptibility (7.1)

$$
M_{\omega}=n \mu\langle\cos \theta\rangle_{\omega}=\chi(\omega) H_{\omega}
$$

one gets

$$
\frac{\chi(\omega)}{\chi_{0}}=\frac{1-\beta / 2 Z_{2}}{1-i \omega \tau_{D}-\beta S_{2} / 2}, \quad \chi_{0}=\frac{n \mu^{2}}{3 T} .
$$

The nondimensional functions entering it, are defined by recurrence relations (8.54), with initial conditions

$$
S_{N}=0, \quad P_{N}=0 .
$$

As already mentioned, the amplitudes of the series terms diminish with the number of the mode with the rate that ensures convergence of the series used in the calculations. Changing parameter $N$, i.e., the highest order of the chain, one can easily control the accuracy of this work. Examples of numerical results are shown below. Solid lines in Figs. 13-15 show the results of calculating $\chi^{\prime \prime}$ with $N \sim 10$. Dashed lines correspond to the effective-field approximation, when the infinite chain of equations (8.46) is restricted to just the pair of first ones.

Note that in the 3D case, the lineshapes at moderate temperatures differ strongly from those obtained for the 2D case (planar dipole rotators), cf. Figs. 12 and 16. Now the low-frequency peak that is due to slow diffusion, does not have a fixed position. Indeed, with the temperature growth, it shifts considerably to the high-frequency side diminishing simultaneously in magnitude, Meanwhile, the high-frequency behaves in the same way as in the case of rotators: decreasing in amplitude, it moves towards the low-frequency end. In result of the mutual approach of the peaks, the absorption line $\chi^{\prime \prime}$ in 3D case may assume a certain asymmetrical shape but never assumes a clear two-peak structure as it happens in 2D case, see Fig. 12.

As it should have been expected, the dynamic susceptibility of an ensemble of magnetic dipoles suspended in a Jeffreys fluid and possessing full set of the rotational degrees of freedom, is similar to the same characteristic of an ensemble of planar rotators. The temperature effect is different, however. Comparing figures 12 and 16, one finds that for the rotators the influence of temperature on the susceptibility spectrum is much stronger. For example, the high-temperature limit, where fast diffusion dominates, is attained already at $T \geq 2 \kappa_{M}$; the low-temperature limit becomes valid at $T<0.1 \kappa_{M}$. For the 3D rotary motion, the region of high-temperature limit is about the same: ; however, the border of low-temperature limit changes substantially, to $T<0.01 \kappa_{M}$, i.e., is an order of magnitude lower than in the ensemble with $2 \mathrm{D}$ rotations.

\section{Conclusions}

Phenomenological theory of Brownian motion in fluids with complex rheology is presented. The rheology of the particle surrounding is modelled with the aid of conventional elements of macroscopic rheology: springs and dampers. These elements might be set in any combination thus endowing the medium with viscoelasticity but always retaining its overall fluidity. By analyzing the possible rheological schemes, it is shown that their variety could be reduced to few simple basic patterns.

For a particle embedded in such a viscoelastic fluid, the Langevin equations are derived and then pertinent diffusion (kinetic) equations are constructed. From analyzing the time dependence of the particle mean-square displacement (MSD), we come to conclusion that a "pure" Maxwell scheme is fundamentally inappropriate for modelling of the particle thermofluctuation motion. In a formal way, 

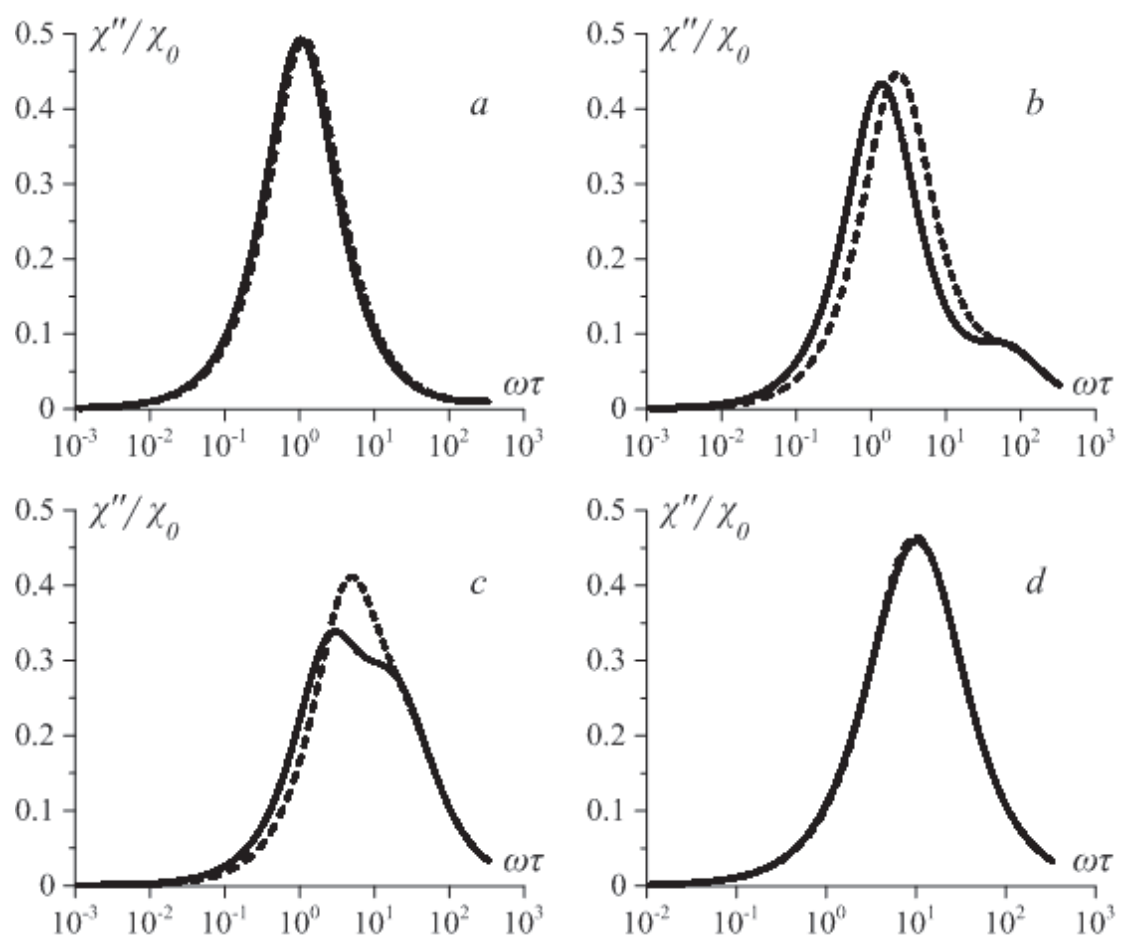

FiguRE 13. Frequency dependence of the imaginary part of dynamic magnetic susceptibility of 3D-rotatable particles for $q=10$; solid lines are obtained via Eq. (8.60), dashed lines are plotted from two-moment approximation of the set $(8.46)\left(M_{2}=0\right)$; the dimensionless temperature is $T / \kappa_{M}=0.01(\mathrm{a}), 0.1(\mathrm{~b}), 0.5(\mathrm{c}), 3(\mathrm{~d})$; the time scale is $\tau=\tau_{D}(1+q)$.

the Maxwell scheme does not have enough elements (material parameters) to avoid essentially unphysical predictions.

A plausible model, however, is just a bit more complicated. It is the Jeffreys fluid, whose "extra" viscosity (damper) turns out to perfectly regularize the problem yielding very reasonable physical results. In particular, the obtained expression that contains two diffusion regimes, fast and slow, and the crossover (dynamic localization) in between, proves to be quite successfully to explain the time dependence of MSD in micellar solutions of the living polymer type.

Some problems related to active microrheology, i.e., sensing the small-scale rheology of complex fluids by detecting the motion of embedded probing microparticles subjected to an external excitation, are solved and analyzed. Namely, the forced orientation diffusion in a dilute ensemble of dipolar particles in the Jeffreys fluid is described in terms of the dynamic magnetic susceptibility. The conditions for revealing both regimes of the particle rotary diffusion as well as the crossover behavior are given, and the manifestations of these mesoscopic effects in the magnetic spectra of the system are described.

The magnetic susceptibility of a simplified model ensemble, where the particles are disk-like and all the dipole moments are confined in a single plane, could be calculated analytically without big effort. The solution of the same problem but for the particles, which possess the full set of orientational degrees of freedom, is much more laborious. Having obtained it for the first time, we perform a comparison of the two models. It turns out that, although the restricted model predicts qualitatively the same behavior, in the quantitative aspect it deviates substantially from that given by the 3D solution. This is important since the new solution is expected to much better reflect the experimental situation. 

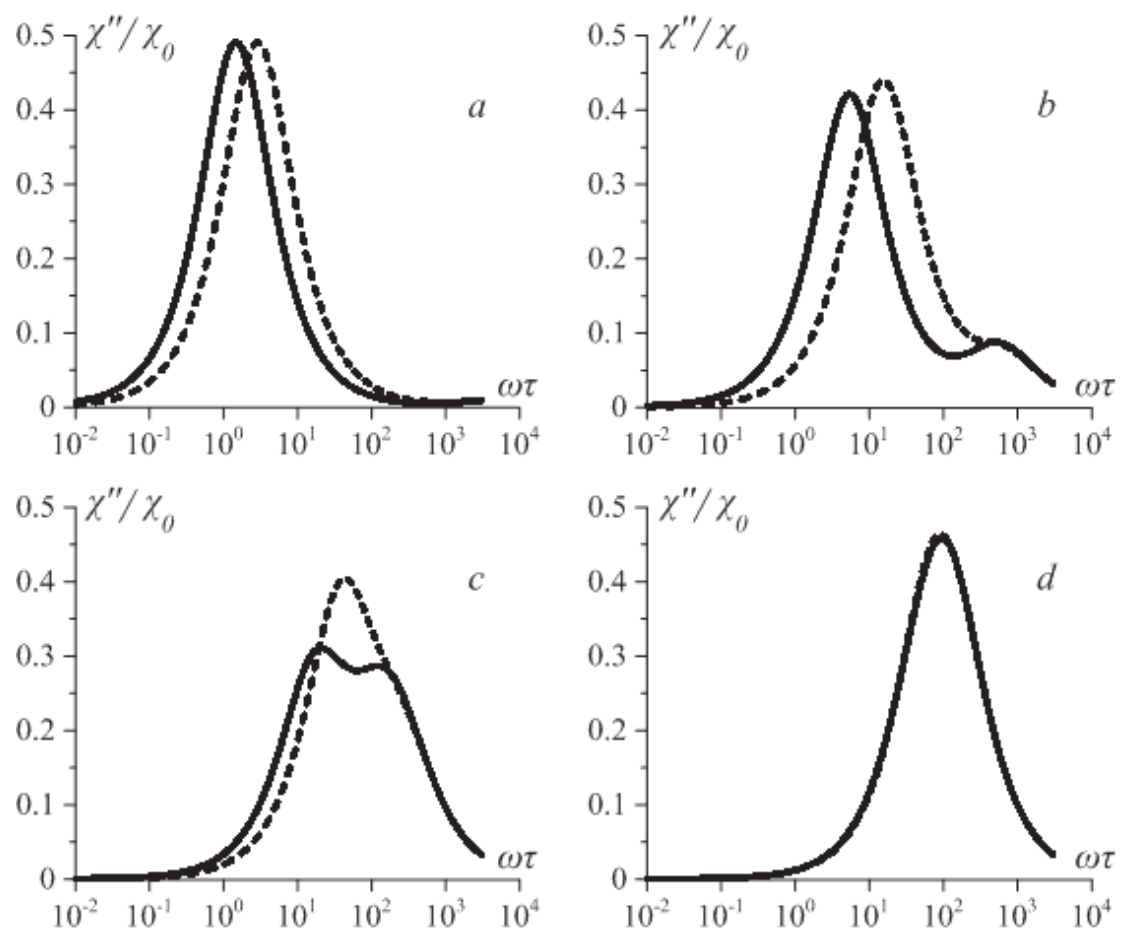

FIGURE 14. Frequency dependence of the imaginary part of dynamic magnetic susceptibility of 3D-rotatable particles for $q=100$; solid lines are obtained via Eq. (8.60), dashed lines are plotted from two-moment approximation of the set (8.46) $\left(M_{2}=0\right)$; the dimensionless temperature is $T / \kappa_{M}=0.01(\mathrm{a}), 0.1(\mathrm{~b}), 0.5(\mathrm{c}), 3(\mathrm{~d})$; the time scale is $\tau=\tau_{D}(1+q)$.

Finally, we note that forced rotational motion of magnetic micro/nanoparticles evolving on a thermofluctuational background nowadays attracts much of attention in connection with magnetic hyperthermia. This term denotes local heating or mechanical excitation generated in a living tissue by embedded magnetic particles subjected to an applied AC field. Since recently, magnetic hyperthermia is becoming a high-tech medical tool. As soon as a realistic theory of dynamic magnetic susceptibility of particle embedded in cell cytosol or adsorbed on cell membranes would be developed, it would immediately become a cornerstone for this technique. Note that the field amplitudes in hyperthermia are quite low due to physiological restrictions. Because of that, the conventional estimate for the local heat generation is based on the simple expression $\omega \chi^{\prime \prime}(\omega)$, which determines the specific loss power due to the particles. As to now, numerous models for $\chi^{\prime \prime}$ are developed whose attention is exclusively focused on the internal magnetic structure and properties of the particles. At the same time, next to nothing is done to account for the rheology of the media, which surrounds the particles and where they move. Most usual, the matrix is assumed to be either a solid body or a linearly viscous (Newtonian) fluid. This seems strange since the rheology of neither of these media even remotely resembles that of the living tissue. With allowance for this fact, we consider our work on modelling $\chi(\omega)$ in viscoelastic magnetic media as a useful step.

\section{Appendix I. Dynamic susceptibility: derivation from kinetic equation}

Formula (7.1) is obtained in the framework of the linear response theory (LRT) and yields the dynamic susceptibility of an ensemble of planar rotators (in-plane dipoles) in a viscoelastic fluid. In this reduced (with respect to angle coordinates) case and in the absence of external field evaluation of the correlator 

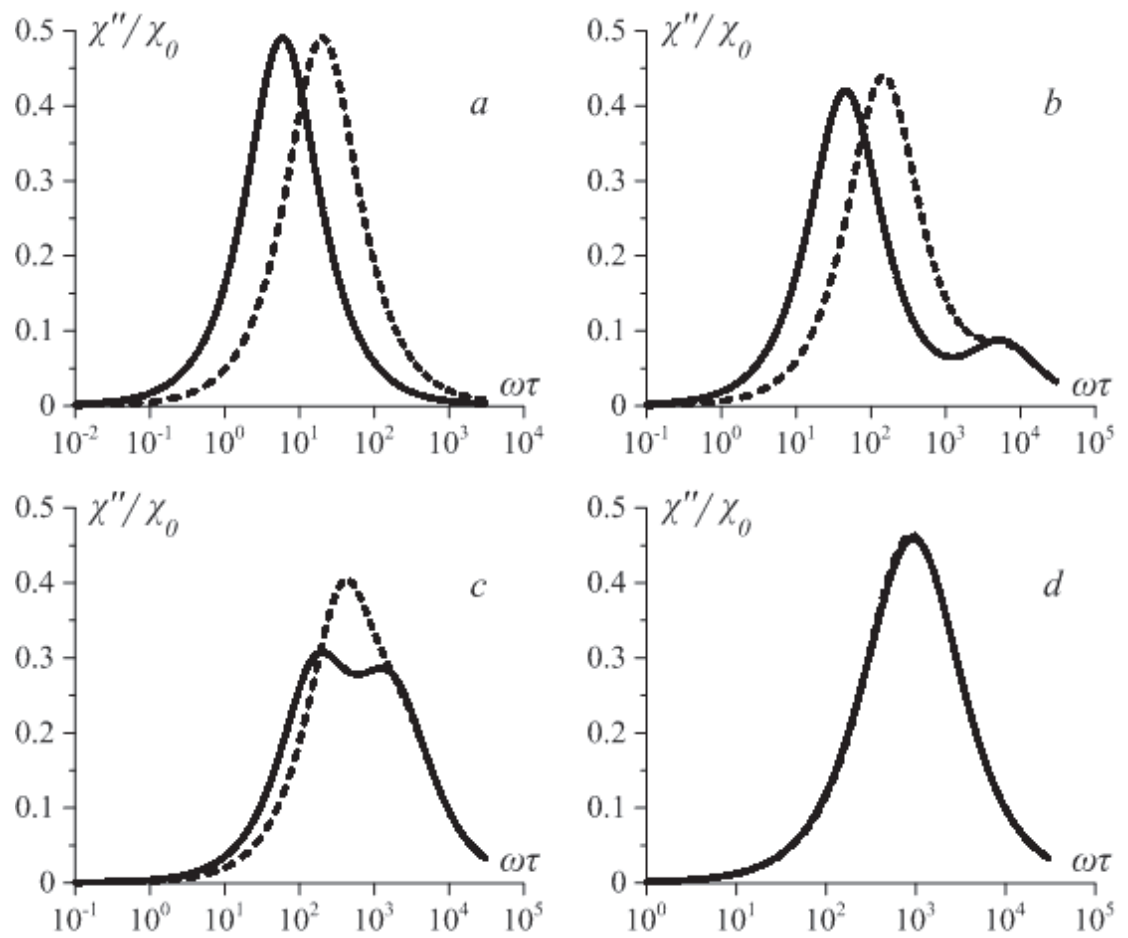

FIgURE 15. Frequency dependence of the imaginary part of dynamic magnetic susceptibility of 3D-rotatable particles for $q=10^{3}$; solid lines are obtained via Eq. (8.60), dashed lines are plotted from two-moment approximation of the set (8.46) $\left(M_{2}=0\right)$; the dimensionless temperature is $T / \kappa_{M}=0.01(\mathrm{a}), 0.1(\mathrm{~b}), 0.5(\mathrm{c}), 3(\mathrm{~d})$; the time scale is $\tau=\tau_{D}(1+q)$.

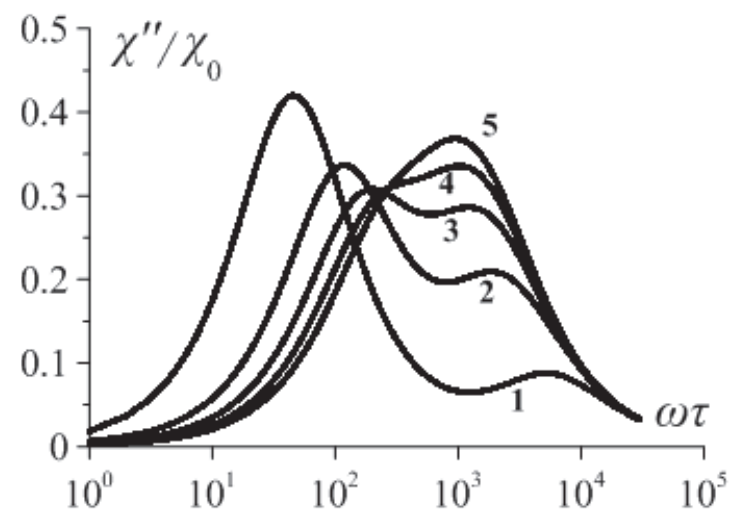

FIgURE 16. Frequency dependence of the imaginary part of dynamic magnetic susceptibility of $3 \mathrm{D}$ rotatable particles at $q=10^{3}$ obtained by formula (8.60); dimensionless temperature $T / \kappa_{M}=0.1,(1), 0.3(2), 0.5(3), 0.7(4), 0.9(5)$.

is sufficiently simple. However, under more complicated conditions, e.g. in the presence of a field or in the case of unrestricted rotation, the LRT is not the most convenient approach. In our view, the moment method, see [24], for example, is more powerful and enables one to solve a number of complicated 
problems. In this section we show how the set of moment equations is derived from the kinetic (FokkerPlanck) equation.

We begin with the set of Langevin equations for non-inertial 1D translational motion of a particle in a Jeffreys fluid and in the presence of external potential. This set has the form

$$
\begin{aligned}
& \left\{\begin{array}{l}
\dot{x}=\frac{1}{\zeta_{N}}\left[-\left(\frac{\partial U}{\partial x}\right)+Q+y_{N}(t)\right], \\
\dot{Q}=-\widetilde{\gamma} Q+\gamma_{N}\left(\frac{\partial U}{\partial x}\right)+\kappa_{M}\left[\frac{y_{M}(t)}{\zeta_{M}}-\frac{y_{N}(t)}{\zeta_{N}}\right],
\end{array}\right. \\
& \left\langle y_{\alpha}(t) y_{\beta}(t+\tau)\right\rangle=2 \delta_{\alpha \beta} T \zeta_{\alpha} \delta(\tau), \quad \alpha, \beta=N, M
\end{aligned}
$$

where $\gamma_{N} \equiv 1 / \tau_{N}=\kappa_{M} / \zeta_{N}$ and $\widetilde{\gamma} \equiv \gamma_{N}+\gamma_{M}=\gamma_{M}(1+q)$. Equation (10.1) differs from the aforeanalyzed Eq. (4.2) only by the presence of potential terms. Using Eq. (10.1) in a similar way as in above for derivation of the kinetic equation, one finds that the single-particle distribution function $W=W(x, Q, t)$ should obey the equation

$$
\begin{aligned}
\frac{\partial}{\partial t} W+\frac{1}{\zeta_{N}} \frac{\partial}{\partial x}\left(Q+T \kappa_{M} \frac{\partial}{\partial Q}\right) W & +\gamma_{N} \frac{\partial}{\partial Q}\left[\left(\frac{\partial U}{\partial x}\right)+T \frac{\partial}{\partial x}\right] W \\
& =\frac{1}{\zeta_{N}} \frac{\partial}{\partial x}\left[\left(\frac{\partial U}{\partial x}\right)+T \frac{\partial}{\partial x}\right] W+\widetilde{\gamma} \frac{\partial}{\partial Q}\left(Q+T \kappa_{M} \frac{\partial}{\partial Q}\right) W
\end{aligned}
$$

which equilibrium solution, as it should be (see (3.23)), is the extended Boltzmann distribution

$$
W_{0}(x, Q) \propto \exp \left[-\frac{1}{T}\left(U(x)+\frac{Q^{2}}{2 \kappa_{m}}\right)\right] .
$$

In particular, formula (10.3) evidences that phase variables $x$ and $Q$ in equilibrium are statistically independent.

In section 4 is shown that for transiting from 1D translational motion to the rotary one about fixed axis, it suffices to replace the spatial coordinate $x$ by the angle variable $\theta$. The potential in the transformed equation (10.2) is the Zeeman energy of the particle magnetic dipole $\boldsymbol{\mu}$ directed under angle $\theta$ to the field, that is

$$
U=-\mu H \cos \theta
$$

The ensemble average value of any function of the system phase variables is evaluated as

$$
\langle\psi(x, Q)\rangle=\int d x \int d Q W(x, Q, t) \psi(x, Q),
$$

where integration spans over all the variation regions of the corresponding coordinates. For plane rotation, these are $0 \leq \theta \leq 2 \pi,-\infty<Q<\infty$, and for the angle variable cyclic boundary conditions should hold: $W(0, Q)=W(2 \pi, Q)$.

Multiplying Eq. (10.2), after having done the replacement $x \rightarrow \theta$, by function $\psi(\theta, Q)$, we perform its term by term integration by parts over the whole phase space of the system. Taking into account the angular cyclicity of $W$ and the homogeneity of boundary conditions with respect to $Q: W(\theta, \pm \infty)=0$, one finds

$$
\begin{aligned}
\frac{\partial}{\partial t}\langle\psi\rangle-\frac{1}{\zeta_{N}}\left\langle Q \frac{\partial \psi}{\partial \theta}\right\rangle+\frac{2 T \kappa_{M}}{\zeta_{N}}\left\langle\frac{\partial^{2} \psi}{\partial Q \partial \theta}\right\rangle-\gamma_{N}\left\langle\frac{\partial U}{\partial \theta} \frac{\partial \psi}{\partial Q}\right\rangle \\
=-\frac{1}{\zeta_{N}}\left\langle\frac{\partial U}{\partial \theta} \frac{\partial \psi}{\partial \theta}\right\rangle+\frac{T}{\zeta_{N}}\left\langle\frac{\partial^{2} \psi}{\partial \theta^{2}}\right\rangle-\widetilde{\gamma}\left\langle Q \frac{\partial \psi}{\partial Q}\right\rangle+\widetilde{\gamma} T \kappa_{M}\left\langle\frac{\partial^{2} \psi}{\partial Q^{2}}\right\rangle
\end{aligned}
$$


It is convenient, to make this equation nondimensional. For that, we choose $t^{\prime}=t / \tau_{D}$ with $\tau_{D}=\zeta_{N} / T$ as the time scale, and make the force nondimensional by way of

$$
z=Q / \sqrt{2 T \kappa_{M}}
$$

After regrouping, the equation for the averages transforms to

$$
\begin{aligned}
\frac{\partial}{\partial t^{\prime}}\langle\psi\rangle-\left\langle\frac{\partial^{2} \psi}{\partial \theta^{2}}\right\rangle+\alpha \beta\left[\left\langle z \frac{\partial \psi}{\partial z}\right\rangle-\frac{1}{2}\left\langle\frac{\partial^{2} \psi}{\partial z^{2}}\right\rangle\right]+\sqrt{2 \beta}\left\langle\left(\frac{\partial}{\partial z}-z\right) \frac{\partial \psi}{\partial \theta}\right\rangle & =\xi\left\langle\sin \theta\left(\sqrt{\frac{\beta}{2}} \frac{\partial}{\partial z}-\frac{\partial}{\partial \theta}\right) \psi\right\rangle
\end{aligned}
$$

here $\alpha=1+q^{-1}, \beta=\kappa_{M} / T$ is inverse temperature, and $\xi=\mu H / T$ nondimensional magnetic field. The dynamic magnetization of the system in the direction of applied field is proportional to $\langle\cos \theta\rangle$, i.e., in Eq. (10.8) one has to set $\psi=\cos \theta$. The presence of differential operator

$$
\hat{Z}=\left(z \frac{\partial}{\partial z}-\frac{1}{2} \frac{\partial^{2}}{\partial z^{2}}\right)
$$

in the left-hand part of Eq. (10.8) and the form of the equilibrium distribution $W_{0} \propto \exp \left(-z^{2}\right)$ point out that, as the functional basis for the moments, it is convenient to take the combinations

$$
\psi_{n}(\theta, z)=H_{n}(z)\left(\cos \theta \cdot \delta_{n, 2 k}+\sin \theta \cdot \delta_{n, 2 k+1}\right), \quad k=0,1,2, \ldots
$$

Here $H_{n}(z)$ are Hermite polynomials [42], which are the eigenfunctions of operator $\hat{Z}$, mutually orthogonal at the whole real axis $-\infty<z<+\infty$ with the weight function $\exp \left(-z^{2}\right)$. Since the sought for linear susceptibility does not depend of the strength of the probing field $H$, the averaging in the right-hand side of equation (10.8) should be done with the equilibrium distribution function $W_{0} \propto \exp \left(-z^{2}\right)$. To obtain the chain of moment equations, we use the relations connecting Hermite polynomials of different order [42]:

$$
\begin{gathered}
\left(z \frac{d}{d z}-\frac{1}{2} \frac{d^{2}}{d z^{2}}\right) H_{n}(z)=n H_{n}(z), \quad z H_{n}=\frac{1}{2}\left(H_{n+1}+2 n H_{n-1}\right), \\
\frac{d}{d z} H_{n}=2 n H_{n-1} \quad \int_{-\infty}^{\infty} d z e^{-Z^{2}} H_{n} H_{m}=2^{n} n ! \sqrt{\pi} \delta_{n m}, \\
H_{n}=(-1)^{n} e^{z^{2}} \frac{d^{n}}{d z^{n}} e^{-z^{2}}, \quad H_{0}=1, \quad H_{1}=2 z, \quad H_{2}=4 z^{2}-2, \ldots
\end{gathered}
$$

With the aid of these formulas, the set of equations for the moments $M_{n}=\left\langle\psi_{n}\right\rangle$ takes the form

$$
\begin{aligned}
& \left(\partial_{t^{\prime}}+1\right) M_{0}+\sqrt{\frac{1}{2} \beta} M_{1}=\frac{1}{2} \xi \\
& \left(\partial_{t^{\prime}}+1+\alpha \beta\right) M_{1}+\sqrt{2 \beta} M_{0}-\sqrt{\frac{1}{2} \beta} M_{2}=\sqrt{\frac{1}{2} \beta} \xi \\
& \ldots \ldots \\
& \left(\partial_{t^{\prime}}+\Gamma_{k}\right) M_{k}+(-1)^{k}\left(\sqrt{\frac{1}{2} \beta} M_{k+1}-k \sqrt{2 \beta} M_{k-1}\right)=0, \\
& \Gamma_{k}=1+\alpha \beta k, \quad k=2,3, \ldots, \quad t^{\prime}=t / \tau_{D} .
\end{aligned}
$$

The linear response to a harmonic excitation $\xi_{t}=\xi_{\omega} \exp (-i \omega t)$ has the same frequency, so differential equations (10.11) turn into an algebraic one upon obvious replacement $\partial_{t^{\prime}} \rightarrow-i \omega \tau_{D}$. Solution of (10.11) 
goes in two steps. First, the homogeneous part of the set is solved, which is used then for evaluation, by means of the first two equations, of the sought for function $\langle\cos \theta\rangle_{\omega}=M_{0}(\omega)$. The homogeneous part of the set obeys a three-term recurrence equation

$$
\begin{aligned}
& Q_{k}^{-} M_{k-1}+Q_{k} M_{k}+Q_{k}^{+} M_{k+1}=0, \\
& Q_{k}=\Gamma_{k}-i \omega \tau_{D}, \quad Q_{k}^{+}=(-1)^{k} \sqrt{\beta / 2}, \quad Q_{k}^{-}=(-1)^{k+1} k \sqrt{2 \beta} .
\end{aligned}
$$

For the sweeping coefficient $S_{k}$ defined as

$$
M_{k}=S_{k} M_{k-1}
$$

the recurrence relation yields

$$
\begin{gathered}
S_{k}=-\frac{Q_{k}^{-}}{Q_{k}+Q_{k}^{+} S_{k+1}}=\frac{(-1)^{k} k \sqrt{2 \beta}}{\widetilde{\Gamma}_{k}+(-1)^{k} \sqrt{\beta / 2} S_{k+1}}=\frac{(-1)^{k} k \sqrt{2 \beta}}{\widetilde{\Gamma}_{k}-\frac{(k+1) \beta}{\widetilde{\Gamma}_{k+1}-\frac{(k+2) \beta}{\widetilde{\Gamma}_{k+2}-\ldots}}} \\
\widetilde{\Gamma}_{k}=\Gamma_{k}-i \omega \tau_{D}
\end{gathered}
$$

The emerging chain fraction should be truncated at a certain step $N$ using the fact that the moment magnitudes go down with the number growth. This evidences that parameter $N$, i.e., the number of the moments used (sweeping "depth"), determines the calculation accuracy.

With the aid of the sweeping coefficient

$$
S_{2}=\frac{2 \sqrt{\beta}}{\widetilde{\Gamma}_{2}-\frac{3 \beta}{\widetilde{\Gamma}_{3}-\ldots}}
$$

We eliminate the second-order moment from the second equation of the set (10.11) and, thus, get a simple set

$$
\begin{aligned}
& \widetilde{\Gamma}_{0} M_{0}+\sqrt{\frac{1}{2} \beta} M_{1}=\frac{1}{2} \xi \\
& \left(\widetilde{\Gamma}_{1}-\sqrt{\frac{1}{2} \beta} S_{2}\right) M_{1}+\sqrt{2 \beta} M_{0}=\sqrt{\frac{1}{2} \beta} \xi
\end{aligned}
$$

whose solution we write in the form

$$
M_{0} \equiv\langle\cos \theta\rangle_{\omega}=\frac{\xi}{2} \frac{1}{1-\frac{i \omega \tau_{D}}{1-S_{1}}}, \quad S_{1}=\frac{\beta}{\widetilde{\Gamma}_{1}-\frac{2 \beta}{\widetilde{\Gamma}_{2}}-\frac{3 \beta}{\widetilde{\Gamma}_{3}-\ldots}} .
$$

This infinite fraction is convenient for numerical calculations as it obeys a simple recurrence relation

$$
S_{k}=\frac{k \beta}{\widetilde{\Gamma}_{k}-S_{k+1}}, \quad S_{N}=0
$$

Magnetization of an ensemble of non-interacting particles in the linear approximation is

$$
M_{\omega}=n \mu\langle\cos \theta\rangle_{\omega}=\chi(\omega) H_{\omega}
$$

with the dynamic susceptibility

$$
\frac{\chi(\omega)}{\chi_{0}}=\frac{2}{\xi} M_{\omega}=\frac{1}{1-\frac{i \omega \tau_{D}}{1-S_{1}}}, \quad \chi_{0}=\frac{n \mu^{2}}{2 T}
$$


Remarkably, expressions (10.20) and (7.4), which determine one and the same physical quantity, look drastically different. However, this difference is just formal: the calculations show that both formulas render entirely coinciding results under any values of material parameters.

Expression (10.20) easily yields the correct asymptotics in the limiting cases of strong $\beta \rightarrow \infty$ and weak $\beta \rightarrow 0$ dynamic elasticity of the carrier medium. For example, at low elasticity $\left(S_{1} \simeq \beta \rightarrow 0\right)$ Eq. (10.20) gives

$$
\left.\frac{\chi(\omega)}{\chi_{0}}\right|_{\beta \rightarrow 0} \simeq \frac{1}{1-i \omega \tau_{D}}, \quad \tau_{D}=\frac{\zeta_{N}}{T},
$$

proving that in this case the relaxation mostly goes via fast diffusion. In the other limit, strong elasticity, from Eq. (10.20) one has

$$
\left.S_{1}\right|_{\beta \rightarrow \infty} \simeq \frac{\beta}{T}=\frac{\beta}{\left(1-i \omega \tau_{D}+\alpha \beta\right)} \simeq \frac{1}{\alpha}=\frac{q}{1+q} .
$$

Substitution of this formula to Eq. (10.20) once again yields the Debye-type susceptibility but with the rate that is $q$ times slower as it should be for slow diffusion.

Therefore, the moment method, as one would expect, renders that same results as LRT. In this section, we derived the chain of equations using the kinetic equation. Meanwhile, the same chain could be obtained directly from the initial Langevin equations, circumventing the probabilistic kinetic equation. This approach is presented in Appendix II because only by this way, see section 8, we are able to obtain analytic expression for the dynamic susceptibility of suspension of dipolar particles in a Jeffreys fluid under full rotary freedom of the particles. It seems quite probable, that other problems, more complicated mathematically, e.g. the susceptibility in the presence of a constant bias field or the nonlinear (quadratic) susceptibility necessary to analyze birefringence, also could be successfully solved within this approach.

\section{Appendix II. Dynamic susceptibility: derivation from Langevin equations}

Replacement $x \rightarrow \theta$ in equation (10.1) and substitution there the dipolar potential (10.4)give the set of Langevin equations describing non-inertial approximation of 2D orientational motion:

$$
\left\{\begin{array}{l}
\dot{\theta}=\frac{1}{\zeta_{N}}\left[-\mu H \sin \theta+Q+y_{N}(t)\right], \\
\dot{Q}=-\widetilde{\gamma} Q+\kappa_{M} \gamma_{H} \sin \theta+\kappa_{M}\left[\frac{y_{M}(t)}{\zeta_{M}}-\frac{y_{N}(t)}{\zeta_{N}}\right], \quad \gamma_{H} \equiv \frac{\mu H}{\zeta_{N}} .
\end{array}\right.
$$

Transforming it by introducing nondimensional retarded friction force and new random forces, one gets:

$$
\left\{\begin{array}{l}
\dot{\theta}=-\gamma_{H} \sin \theta+\gamma_{N} \widetilde{Q}+u(t), \\
\dot{\widetilde{Q}}=-\widetilde{\gamma} \widetilde{Q}+\gamma_{H} \sin \theta+\widetilde{u}(t)
\end{array}\right.
$$

here

$$
\widetilde{Q} \equiv \frac{Q}{\kappa_{M}}, \quad \widetilde{\gamma} \equiv \kappa_{M}\left(\frac{1}{\zeta_{M}}+\frac{1}{\zeta_{N}}\right), \quad u \equiv \frac{y_{N}(t)}{\zeta_{N}}, \quad \widetilde{u} \equiv \frac{y_{M}(t)}{\zeta_{M}}-\frac{y_{N}(t)}{\zeta_{N}}
$$

and

$$
\begin{aligned}
\langle u(t) u(t+\tau)\rangle & =\left(2 T / \zeta_{N}\right) \delta(\tau), \\
\langle u(t) \widetilde{u}(t+\tau)\rangle & =-\left(2 T / \zeta_{N}\right) \delta(\tau), \\
\langle\widetilde{u}(t) \widetilde{u}(t+\tau)\rangle & =2 T\left(1 / \zeta_{N}+1 / \zeta_{M}\right) \delta(\tau) .
\end{aligned}
$$

In below, the tildes are omitted for brevity. 
For the observable quantity $\langle\cos \theta\rangle$, according to the first of equations (11.1) one has

$$
\partial_{t}\langle\cos \theta\rangle=\gamma_{H}\left\langle\sin ^{2} \theta\right\rangle-\gamma_{N}\langle Q \sin \theta\rangle-\langle u(t) \sin \theta\rangle .
$$

To find the linear response, the first term in the right-hand part of this equation should be averaged over the equilibrium state, that yields $\left\langle\sin ^{2} \theta\right\rangle_{0}$. In equation (11.3), the correlator with the random force is calculated by the already described procedure, where the function is replaced by its singular part

$$
\delta(\sin \theta)=\lim _{\delta \rightarrow 0} \int_{t-\delta}^{t} d t^{\prime} \cos \theta\left(t^{\prime}\right) u\left(t^{\prime}\right) \equiv \hat{I}\left(u^{\prime} \cos \theta^{\prime}\right) .
$$

Here and below, the prime means that the function has argument $t^{\prime}$. With allowance for the properties of white noise, integration yields for the sought for correlation function:

$$
\langle u(t) \sin \theta\rangle=\hat{I}\left\langle u u^{\prime} \cos \theta^{\prime}\right\rangle=\frac{T}{\zeta_{N}}\langle\cos \theta\rangle .
$$

We remark that numeric coefficient here (cf. (4.6)) is two times smaller. This occurs because integration here is performed over the half of the definition domain of $\delta$-function. The resulting equation for the observable quantity is:

$$
\left(\partial_{t}+\gamma_{D}\right)\langle\cos \theta\rangle=\frac{1}{2} \gamma_{H}-\gamma_{N}\langle Q \sin \theta\rangle, \quad \gamma_{D}=1 / \tau_{D}=T / \zeta_{N} .
$$

This equation contains additional moment $\langle Q \sin \theta\rangle$ induced by the viscoelasticity of the carrier medium. Using the set of stochastic equations (11.1), we get for this function equation

$$
\left(\partial_{t}+\widetilde{\gamma}\right)\langle Q \sin \theta\rangle=\gamma_{H}\left(\left\langle\sin ^{2} \theta\right\rangle-\langle\sin \theta \cos \theta Q\rangle\right)
$$

$$
+\gamma_{N}\left\langle Q^{2} \cos \theta\right\rangle+\langle\widetilde{u} \sin \theta\rangle+\langle u Q \cos \theta\rangle .
$$

As before, the field term is averaged over the equilibrium distribution.

Evaluating the diffusion component in the equation for the moment, which is of the first order in $Q$, we get with the aid of the set of Langevin equation (11.1):

$$
\langle\widetilde{u} \sin \theta\rangle+\langle u Q \cos \theta\rangle=\hat{I}\left\langle\widetilde{u} u^{\prime} \cos \theta+u \widetilde{u}^{\prime} \cos \theta-u u^{\prime} Q \sin \theta\right\rangle
$$

$$
=-2 \gamma_{D}\langle\cos \theta\rangle-\gamma_{D}\langle Q \sin \theta\rangle \text {. }
$$

Then the sought for equation takes the form

$$
\left(\partial_{t}+\widetilde{\gamma}+\gamma_{D}\right)\langle Q \sin \theta\rangle=\frac{1}{2} \gamma_{H}-2 \gamma_{D}\langle\cos \theta\rangle+\gamma_{N}\left\langle Q^{2} \cos \theta\right\rangle .
$$

As it should be, the first-order equation includes the moment of second order, and this chaining of equations holds in any order. To simplify presentation of these equations, one needs to pass to the set of orthogonalized moments.

They are constructed in the following way. The non-equilibrium distribution function is expanded as

$$
W(\theta, Q, t)=W_{0}(Q)\left[1+b_{0}(t) \psi_{0}(\theta)+b_{1}(t) \psi_{1}(\theta, Q)+b_{2}(t) \psi_{2}(\theta, Q)+\ldots\right],
$$

for which normalizing conditions require

$$
\left\langle\psi_{k}\right\rangle_{0}=0, \quad k=0,1, \ldots
$$

where index 0 indicates averaging with equilibrium distribution $W_{0}$. Imposing orthogonality conditions on expansion (11.10), one gets

$$
\left\langle\psi_{0}(\theta)\right\rangle=b_{0}(t)\left\langle\psi_{0}^{2}\right\rangle_{0}, \quad b_{0}(t)=\frac{\left\langle\psi_{0}\right\rangle}{\left\langle\psi_{0}^{2}\right\rangle_{0}}, \quad\left\langle\psi_{0} \psi_{k}\right\rangle=0, \quad k=1,2, \ldots
$$


and

$$
b_{n}(t)=\frac{\left\langle\psi_{n}\right\rangle}{\left\langle\psi_{n}^{2}\right\rangle_{0}} \quad \text { for } \quad\left\langle\psi_{n} \psi_{k}\right\rangle_{0}=0, \quad k=n+1, \ldots
$$

In the problem under consideration, the averaging procedure is defined as

$$
\langle\ldots\rangle_{0}=\frac{1}{2 \pi \sqrt{2 \pi \widetilde{T}}} \int_{0}^{2 \pi} d \theta \int_{-\infty}^{\infty} d Q e^{-\frac{Q^{2}}{2 \widetilde{T}}}(\ldots),
$$

and the first basis functions have the form

$$
\psi_{0}=\cos \theta, \quad \psi_{1}=Q \sin \theta, \quad \widetilde{\psi}_{2}=Q^{2} \cos \theta .
$$

For these function the normalizing condition holds identically. The product $\left\langle\psi_{0} \psi_{1}\right\rangle_{0}$, turns to zero due to the same property. Besides that, $\left\langle\psi_{0} \psi_{1}\right\rangle_{0}$ contains an odd function of $Q$, which by itself turns the integral to zero. This ensures orthogonality of all the even (in $Q$ and $\theta$ ) functions to the odd ones. Therefore, orthogonalization should be performed only inside the sets of the basis functions. For example,

$$
\left\langle\psi_{0} \widetilde{\psi}_{2}\right\rangle_{0}=\left\langle\cos ^{2} \theta Q^{2}\right\rangle_{0}=\frac{1}{2} \widetilde{T} \neq 0 .
$$

Let us renormalize the basis function of index 2 as

$$
\psi_{2}=\left(Q^{2}+A\right) \cos \theta,
$$

and then require its orthogonality:

$$
\left\langle\psi_{0} \psi_{2}\right\rangle_{0}=\left\langle\cos ^{2} \theta\left(Q^{2}+A\right)\right\rangle_{0}=\frac{1}{2}\left(\left\langle Q^{2}\right\rangle_{0}+A\right)=0 .
$$

Evaluating coefficient $A$, one finds the "corrected" function

$$
\psi_{2}=\left(Q^{2}-\widetilde{T}\right) \cos \theta=\widetilde{T}\left(\widetilde{z}^{2}-1\right) \cos \theta, \quad \widetilde{z}^{2} \equiv Q^{2} / \widetilde{T} .
$$

Performing similar operations on all the other functions in expansion (11.10), we arrive at the representations

$$
\begin{aligned}
& \psi_{3}=(\widetilde{T})^{3 / 2}\left(\widetilde{z}^{3}-3 \widetilde{z}\right) \sin \theta, \\
& \psi_{4}=\widetilde{T}^{2}\left(\widetilde{z}^{4}-6 \widetilde{z}^{2}+3\right) \cos \theta, \\
& \ldots \\
& \psi_{k}=(\widetilde{T})^{k / 2} \widetilde{H}_{k}(\widetilde{z})\left(\cos \theta \delta_{k, 2 n}+\sin \theta \delta_{n, 2 k+1}\right), \quad n=2,3, \ldots
\end{aligned}
$$

Here $\widetilde{H}_{k}(z)$ are the so-called mathematical Hermite polynomials (widely used in mathematical statistics) [42] which are defined as

$$
\widetilde{H}_{k}(\widetilde{z})=(-1)^{k} e^{\widetilde{z}^{2} / 2} \frac{d^{k}}{d \widetilde{z}^{k}} e^{-\widetilde{z}^{2} / 2} .
$$

They transform into "physical" Hermit polynomials under a simple re-scaling. The connection follows from comparison of definitions (11.21) and (10.10):

$$
H_{k}(z)=2^{k / 2} \widetilde{H}_{k}(\sqrt{2} z) .
$$

In the orthogonalized representation, equation (11.8) for the first moment writes as

$$
\begin{aligned}
& \left(\partial_{t}+\widetilde{\gamma}+\gamma_{D}\right) \widetilde{M}_{1}=\frac{1}{2} \gamma_{H}-\gamma_{D} \widetilde{M}_{0}+\gamma_{N} \widetilde{M}_{2}, \\
& \widetilde{M}_{0}=\langle\cos \theta\rangle, \quad \widetilde{M}_{1}=\langle Q \sin \theta\rangle, \quad \widetilde{M}_{k}=\left\langle\psi_{k}\right\rangle, \ldots
\end{aligned}
$$


As a next step, one needs to derive the equation for the second orthogonal moment. The equation for the non-orthogonalized one is

$$
\partial_{t}\left\langle Q^{2} \cos \theta\right\rangle=2\langle Q \dot{Q} \cos \theta\rangle-\left\langle Q^{2} \dot{\theta} \sin \theta\right\rangle .
$$

With the aid of the Langevin set (11.1), it transforms to

$$
\begin{aligned}
\left(\partial_{t}+2 \widetilde{\gamma}\right)\left\langle Q^{2} \cos \theta\right\rangle=\gamma_{H}\left(2\langle Q \cos \theta \sin \theta\rangle_{0}\right. & \left.+\left\langle Q^{2} \sin ^{2} \theta\right\rangle_{0}\right) \\
& -\gamma_{N}\left\langle Q^{3} \sin \theta\right\rangle+2\langle\widetilde{u}(t) Q \cos \theta\rangle-\left\langle u(t) Q^{2} \sin \theta\right\rangle .
\end{aligned}
$$

Its diffusion part (induced by the random forces) expands as

$$
\begin{array}{r}
2\langle\widetilde{u} Q \cos \theta\rangle-\left\langle u Q^{2} \sin \theta\right\rangle=\hat{I}\left\langle 2 \widetilde{u} \widetilde{u}^{\prime} \cos \theta-2 \widetilde{u} u^{\prime} Q \sin \theta^{\prime}-2 u \widetilde{u}^{\prime} Q \sin \theta-u u^{\prime} Q^{2} \cos \theta^{\prime}\right\rangle \\
=\frac{2 T}{\zeta_{N}}\left(1+q^{-1}\right)\langle\cos \theta\rangle+\frac{4 T}{\zeta_{N}}\langle Q \sin \theta\rangle-\frac{T}{\zeta_{N}}\left\langle Q^{2} \cos \theta\right\rangle .
\end{array}
$$

In result, equation (11.25) takes the form

$$
\begin{aligned}
\left(\partial_{t}+2 \widetilde{\gamma}+\gamma_{D}\right)\left\langle Q^{2} \cos \theta\right\rangle=\gamma_{H} \frac{\widetilde{T}}{2}-\gamma_{N}\left\langle Q^{3} \sin \theta\right\rangle \quad & \\
& +2 \gamma_{D}\left(1+q^{-1}\right)\langle\cos \theta\rangle+4 \gamma_{D}\langle Q \sin \theta\rangle .
\end{aligned}
$$

It contains three moments: $\widetilde{M}_{0}, \widetilde{M}_{1},\left\langle Q^{3} \sin \theta\right\rangle$, and the external probing field proportional to $\gamma_{H}$. Orthogonalizing Eq. (11.27) using relations (11.20), we arrive at

$$
\left(\partial_{t}+2 \widetilde{\gamma}+\gamma_{D}\right) \widetilde{M}_{2}=2 \gamma_{D} \widetilde{M}_{1}-\gamma_{N} \widetilde{M}_{3}
$$

In the same way, after rather cumbersome calculations two more equations are derived, for the orthogonalized moments $\widetilde{M}_{3}$ and $\widetilde{M}_{4}$. Collecting all the obtained equations, one has

$$
\begin{aligned}
& \left(\partial_{t}+\gamma_{D}\right) \widetilde{M}_{0}=\frac{1}{2} \gamma_{H}-\gamma_{N} \widetilde{M}_{1}, \\
& \left(\partial_{t}+\widetilde{\gamma}+\gamma_{D}\right) \widetilde{M}_{1}=\frac{1}{2} \gamma_{H}-\gamma_{D} \widetilde{M}_{0}+\gamma_{N} \widetilde{M}_{2}, \\
& \left(\partial_{t}+2 \widetilde{\gamma}+\gamma_{D}\right) \widetilde{M}_{2}=2 \gamma_{D} \widetilde{M}_{1}-\gamma_{N} \widetilde{M}_{3} \\
& \left(\partial_{t}+3 \widetilde{\gamma}+\gamma_{D}\right) \widetilde{M}_{3}=-3 \gamma_{D} \widetilde{M}_{2}+\gamma_{N} \widetilde{M}_{4} \\
& \left(\partial_{t}+4 \widetilde{\gamma}+\gamma_{D}\right) \widetilde{M}_{4}=4 \gamma_{D} \widetilde{M}_{3}-\gamma_{N} \widetilde{M}_{5} .
\end{aligned}
$$

This set suffices for determining by induction the general form of the chain. As all the equations of the order greater than two are homogeneous, one gets an easily verified recurrence relation

$$
\left(\partial_{t}+k \gamma+\gamma_{D}\right) \widetilde{M}_{k}=(-1)^{k}\left(k \gamma_{D} \widetilde{M}_{k-1}-\gamma_{N} \widetilde{M}_{k+1}\right), \quad k \geq 2,3, \ldots
$$

In conclusion. The here derived - directly from the set of Langevin equatons - chain of moment equations is equivalent to that constructed in Appendix I, which is obtained on the base of kinetic equation. To prove that, one needs just to apply definition (11.20) for the moments $\widetilde{M}_{k}$ :

$$
\begin{aligned}
& \widetilde{M}_{k}=\left\langle\psi_{k}\right\rangle=\widetilde{T}^{k / 2}\left\langle\widetilde{H}_{k}(\widetilde{z})\left(\delta_{2 n, k} \cdot \cos \theta+\delta_{2 n+1, k} \cdot \sin \theta\right)\right\rangle, \\
& \widetilde{z}=Q / \sqrt{\kappa_{M} T}=\sqrt{2} z,
\end{aligned}
$$

and take into account relation (11.22) between Hermite polynomials of two kinds. There are certain methodical differences, however. Using kinetic equation as a starting point, it is possible to determine 
explicitly the set of orthogonal basis functions for arbitrary order and to get a recurrence formula for all the moments. The Langevin equations method admits only a step-by-step procedure for constructing orthogonal basis functions, so that the general form could be deduced just by induction. However, remarkably, right this step-wise procedure turns out to be the advantage of the method when applied to complicated problems. One example is given in detail in section 8 , where the dynamic susceptibility of an ensemble of dipolar particles with the full set of orientational degrees of freedom is evaluated analytically.

The work was supported by RFBR Grant No. 14-02-96003 and project MIG S26/617 from the Ministry of Education and Science of Perm Region.

\section{References}

[1] M. L. Gardel, M. T. Valentine, D. A. Weitz.Microrheology. In: Microscale Diagnostic Techniques, K. Breuer, Ed. New York: Springer, 2005.

[2] T. A. Waigh T. A.Microrheology of complex fluids. Reports on Progress in Physics, 68 (2005), 685-742.

[3] A. Yao, M. Tassieri, M. Padgett, J. Cooper. Microrheology with optical tweezers. Lab on Chip, 9 (2009), $2568-2575$.

[4] P.-H. Wu, C. M. Hale, W.-C. Chen, J. S. H. Lee, Y. Tseng, D. Wirtz.High-throughput ballistic injection nanorheology to measure cell mechanics. Nature Protocols, 7 (2012), 155-170.

[5] S. Dutz, R. Hergt.Magnetic nanoparticle heating and heat transfer on a microscale: Basic principles, realities and physical limitations of hyperthermia for tumour therapy. Int. J. Hyperthermia, 29 (2013), 790-800.

[6] S. Dutz, R. Hergt.Magnetic particle hyperthermia - a promising tumour therapy? Nanotechnology, 25 (2014), Art. no. 252001 (28 pages).

[7] E. Amstad, J. Kohlbrecher, E. Müller, T. Schweizer, M. Textor, E. Reimhult. Triggered release from liposomes through magnetic actuation of iron oxide nanoparticle containing membranes. Nano Letters, 11 (2011), 1664-1670.

[8] R. Deckers, C. Debeissat, P. Y. Fortin, C. T. W. Moonen, F. Couillaud.Arrhenius analysis of the relationship between hyperthermia and Hsp70 promoter activation: A comparison between ex vivo and in vivo data. Int. J. Hyperthermia, 28 (2012), 441-450.

[9] M. B. Bannwarth, S. Ebert, M. Lauck, U. Ziener, S. Tomcin, G. Jakob, K. Münnemann, V. Mailänder, A. Musyanovych, K. Landfester. Tailor-made nanocontainers for combined magnetic-field-induced release and MRI. Macromolecular Bioscience, 14 (2014), 1205-1214.

[10] B. Mehdaoui, A. Meffre, J. Carrey, S. Lachaize, L.-M. Lacroix, M. Gougeon, D. Chaudret, M. Respaud. Optimal size of nanoparticles for magnetic hyperthermia: A combined theoretical and experimental study. Advanced Functional Materials, 21 (2011), 4573-4581.

[11] C. Martinez-Boubeta, K. Simeonidis, A. Makridis, M. Angelakeris, O. Iglesias, P. Guardia, A. Cabot, L. Yedra, S. Estradé, E. Peiró, Z. Saghi, P. A. Midgley, I. Conde-Leborán, D. Serantes, D. Baldomir.Learning from nature to improve the heat generation of iron-oxide nanoparticles for magnetic hyperthermia applications. Scientific Reports, 3 (2013), Art. no. 1652 (8 pages).

[12] R. Di Corato R., A. Espinosa, L. Lartigue, M. Tharaud, S. Chat, T. Pellegrino, C. Ménager, F. Gazeau, C. Wilhelm. Magnetic hyperthermia efficiency in the cellular environment for different nanoparticle designs. Biomaterials, 35 (2014), 6400-6411.

[13] Yu. L. Raikher, V. I. Stepanov.Physical aspects of magnetic hyperthermia: Low-frequency ac field absorption in a magnetic colloid. J. Magnetism and Magnetic Materials, 368 (2014), 421-427.

[14] D. Robert, K. Aubertin, J.-C. Bacri, C. Wilhelm.Magnetic nanomanipulations inside living cells compared with passive tracking of nanoprobes to get consensus for intracellular mechanics. Physical Review E, 85 (2012), Art. no. 011905 (9 pages).

[15] E. Roeben, L. Roeder, S. Teusch, M. Effertz, U. K. Deiters, A. M. Schmidt.Magnetic particle nanorheology. Colloid and Polymer Science, 292 (2014), 2013-2023.

[16] A. Ya. Malkin, A. I. Isayev.Rheology: Conceptions, Methods, Applications. Toronto, Chemtech Publishers, 2005.

[17] P. Oswald.Rheophysics: The Deformation and Flow of Matter. Cambridge, Cambridge University Press, 2009.

[18] L. D. Landau, E. M. Lifshitz.Fluid Mechanics. Oxford, Pergamon Press, 1959.

[19] A. Einstein. Über die von der molekularkinetischen Theorie der Wärme geforderte Bewegung von in ruhenden Flüssigkeiten suspendierten Teilchen. Annalen der Physik, 322 (1905), 549-560.

[20] H. Haken.Synergetics. Non-equilibrium Phase Transitions and Self-Organization in Physics, Chemistry and Biology. Berlin, Springer, 1978. 2nd ed.

[21] H. Risken. The Fokker-Planck Equation. Springer Series in Synergetics. 1984. Vol. 18. Ed. H. Haken. Berlin, Springer.

[22] V. I. Klyatskin.Dynamics of Stochastic Systems. Amsterdam, Elsevier, 2005.

[23] Yu. L. Klimontovich.Statistical Theory of Open Systems. Dordrecht, Kluwer, 1995.

[24] W. T. Coffey, Yu. P. Kalmykov. The Langevin Equation. World Scientific, Singapore. 2012. 3rd ed.

[25] P. Langevin.Sur la théorie du mouvement brownien. Comptes Rendus Acad. Sci., (Paris), 146 (1908), $530-533$.

[26] C. Wilhelm, F. Gazeau, J.-C. Bacri.Rotational magnetic endosome microrheology: Viscoelastic architecture inside living cells. Physical Review E., 67 (2003), Art. no. 061908 (12 pages). 
[27] Yu. L. Raikher, V. V. Rusakov.Brownian motion in a Jefferys fluid. J. Experimental and Theoretical Physics, 111 (2010), 883-889.

[28] Yu. L. Raikher, V. V. Rusakov, R. Perzynski.Brownian motion in a viscoelastic medium modelled by a Jeffreys fluid. Soft Matter, 9 (2013), 10857-10865.

[29] L. D. Landau, E. M. Lifshitz.Statistical Physics. New York, Pergamon Press, 1980. 3rd ed. Pt.1.

[30] J. H. van Zanten, K. P. Rufener.Brownian motion in a single relaxation time Maxwell fluid. Physical Review E, 62 (2000), 5389-5396.

[31] F. Cardinaux, L. Cipelletti, F. Scheefold, P. Schurtenberger.Microrheology of giant-micelle solutions. Europhysics Letters, 57 (2002), 738-744.

[32] A. Ochab-Marcinek, R. Holyst.Scale-dependent diffusion of spheres in solutions of flexible and rigid polymers: mean square displacement and autocorrelation function for FCS and DLS measurements. Soft Matter, 7 (2011), 7366-7374.

[33] T. K. Piskorz, A. Ochab-Marcinek. A universal model of restricted diffusion for fluorescence correlation spectroscopy. J. Physical Chemistry B, 118. (2014), 4906-4912.

[34] Yu. L. Raikher, V. V. Rusakov.Dynamic susceptibility of viscoelastic magnetic fluids. J. Experimental and Theoretical Physics, 83 (1996), 988-995.

[35] V. S. Volkov. Theory of Brownian motion in a viscoelastic Maxwell fluid. J. Experimental and Theoretical Physics, 71 (1990), 93-98.

[36] J.-L. Déjardin. Viscoelastic effects on the dynamic susceptibility of a Brownian particle in an external potential. Physical Review E, 58 (1998), 2808-2817.

[37] V. S. Volkov, A. I. Leonov.Non-Markovian Brownian motion in a viscoelastic fluid. J. Chemical Physics, 104 (1996), 5922-5931.

[38] V. S. Volkov, A. I. Leonov.Rotational Brownian motion of axisymmetric particles in a Maxwell fluid. Physical Review E, 64 (2001), Art. no. 051113 (9 pages).

[39] A. Baura, S. Ray, M. K. Sen, B. C. Bag.Study of non-Markovian dynamics of a charged particle in presence of a magnetic field in a simple way. J. Applied Physics, 113 (2013), Art. no. 124905 (11 pages).

[40] P. G. de Gennes.Scaling Concepts in Polymer Physics. Ithaca, Cornell University Press, 1979.

[41] Yu. L. Raikher, V. I. Stepanov.Nonlinear dynamic susceptibilities and field-induced birefringence in magnetic particle assemblies. Advances in Chemical Physics, 129 (2004), 419-588.

[42] M. Abramovitz, I. A. Stegun, Eds.Handbook of Mathematical Functions. New York, Dover, 1965. 